\title{
Comparative transcriptome profiling of amyloid precursor protein family members in the adult cortex
}

Dorothee Aydin ${ }^{1 \dagger}$, Mikhail A Filippov ${ }^{1 \dagger}$, Jakob-Andreas Tschäpe ${ }^{1}$, Norbert Gretz ${ }^{2}$, Marco Prinz ${ }^{3}$, Roland Eils ${ }^{1,4}$, Benedikt Brors ${ }^{4}$ and Ulrike C Müller ${ }^{{ }^{*}}$

\begin{abstract}
Background: The $\beta$-amyloid precursor protein (APP) and the related $\beta$-amyloid precursor-like proteins (APLPS) undergo complex proteolytic processing giving rise to several fragments. Whereas it is well established that $A \beta$ accumulation is a central trigger for Alzheimer's disease, the physiological role of APP family members and their diverse proteolytic products is still largely unknown. The secreted APPs $\alpha$ ectodomain has been shown to be involved in neuroprotection and synaptic plasticity. The $\gamma$-secretase-generated APP intracellular domain (AICD) functions as a transcriptional regulator in heterologous reporter assays although its role for endogenous gene regulation has remained controversial.
\end{abstract}

Results: To gain further insight into the molecular changes associated with knockout phenotypes and to elucidate the physiological functions of APP family members including their proposed role as transcriptional regulators, we performed DNA microarray transcriptome profiling of prefrontal cortex of adult wild-type (WT), APP knockout $\left(\mathrm{APP}^{-/}\right), \mathrm{APLP} 2$ knockout (APLP2 ${ }^{-/}$) and APPs $\alpha$ knockin mice (APP ${ }^{\alpha / \alpha}$ ) expressing solely the secreted APPs $\alpha$ ectodomain. Biological pathways affected by the lack of APP family members included neurogenesis, transcription, and kinase activity. Comparative analysis of transcriptome changes between mutant and wild-type mice, followed by qPCR validation, identified co-regulated gene sets. Interestingly, these included heat shock proteins and plasticity-related genes that were both down-regulated in knockout cortices. In contrast, we failed to detect significant differences in expression of previously proposed AICD target genes including Bace1, Kail, Gsk3b, p53, Tip60, and Vglut2. Only Egfr was slightly up-regulated in APLP2 ${ }^{-/}$mice. Comparison of APP ${ }^{-/}$and $\mathrm{APP}^{\alpha / \alpha}$ with wildtype mice revealed a high proportion of co-regulated genes indicating an important role of the C-terminus for cellular signaling. Finally, comparison of $\mathrm{APLP}^{-1-}$ on different genetic backgrounds revealed that backgroundrelated transcriptome changes may dominate over changes due to the knockout of a single gene.

Conclusion: Shared transcriptome profiles corroborated closely related physiological functions of APP family members in the adult central nervous system. As expression of proposed AICD target genes was not altered in adult cortex, this may indicate that these genes are not affected by lack of APP under resting conditions or only in a small subset of cells.

\footnotetext{
* Correspondence: u.mueller@urz.uni-hd.de

† Contributed equally

'Department of Bioinformatics and Functional Genomics, Institute of Pharmacy and Molecular Biotechnology, Heidelberg University, Im Neuenheimer Feld 364, D-69120 Heidelberg, Germany

Full list of author information is available at the end of the article
} 


\section{Background}

Despite its key role in Alzheimer's disease (AD) pathogenesis, the physiological functions of the $\beta$-amyloid precursor protein (APP) and its close homologue, the $\beta$ amyloid precursor-like protein 2 (APLP2), are still poorly understood. This is due to two major problems complicating the in vivo analysis. i) APP is subject to complex proteolytical processing and ii) APP is part of a gene family with partially overlapping functions.

APP is a type I transmembrane protein, and processing (see Figure 1a) is initiated either by $\alpha$-secretase cleavage within the $A \beta$ region, or by $\beta$-secretase (BACE) cleavage at the $\mathrm{N}$-terminus of $\mathrm{A} \beta$, leading to the secretion of large soluble ectodomains, termed APPs $\alpha$ and APPs $\beta$ respectively. Subsequent $\gamma$-secretase processing of the C-terminal fragments $(\beta \mathrm{CTF}$, or $\alpha \mathrm{CTF})$ results in the production of secreted $\mathrm{A} \beta, \mathrm{p} 3$ and the APP intracellular domain (AICD). Both APLPs are similarly processed by the same secretases. It is evident that APP/ APLPs are highly complex molecules, that may exert important functions as unprocessed cell surface molecules (APP-FL) as well as functions mediated by their diverse proteolytic fragments. APP processing is highly reminiscent to that of Notch with $\gamma$-secretase-mediated release of the Notch intracellular domain (NICD) triggering the translocation of NICD to the nucleus. This results in transcriptional regulation of defined target genes involved in e.g. neuronal differentiation. Thus, a similar functional role for AICD (and the related intracellular fragments of APLPs, termed ALID1 and ALID2) as transcriptional regulator has been proposed [1]. Indeed, AICD has been shown to translocate to the

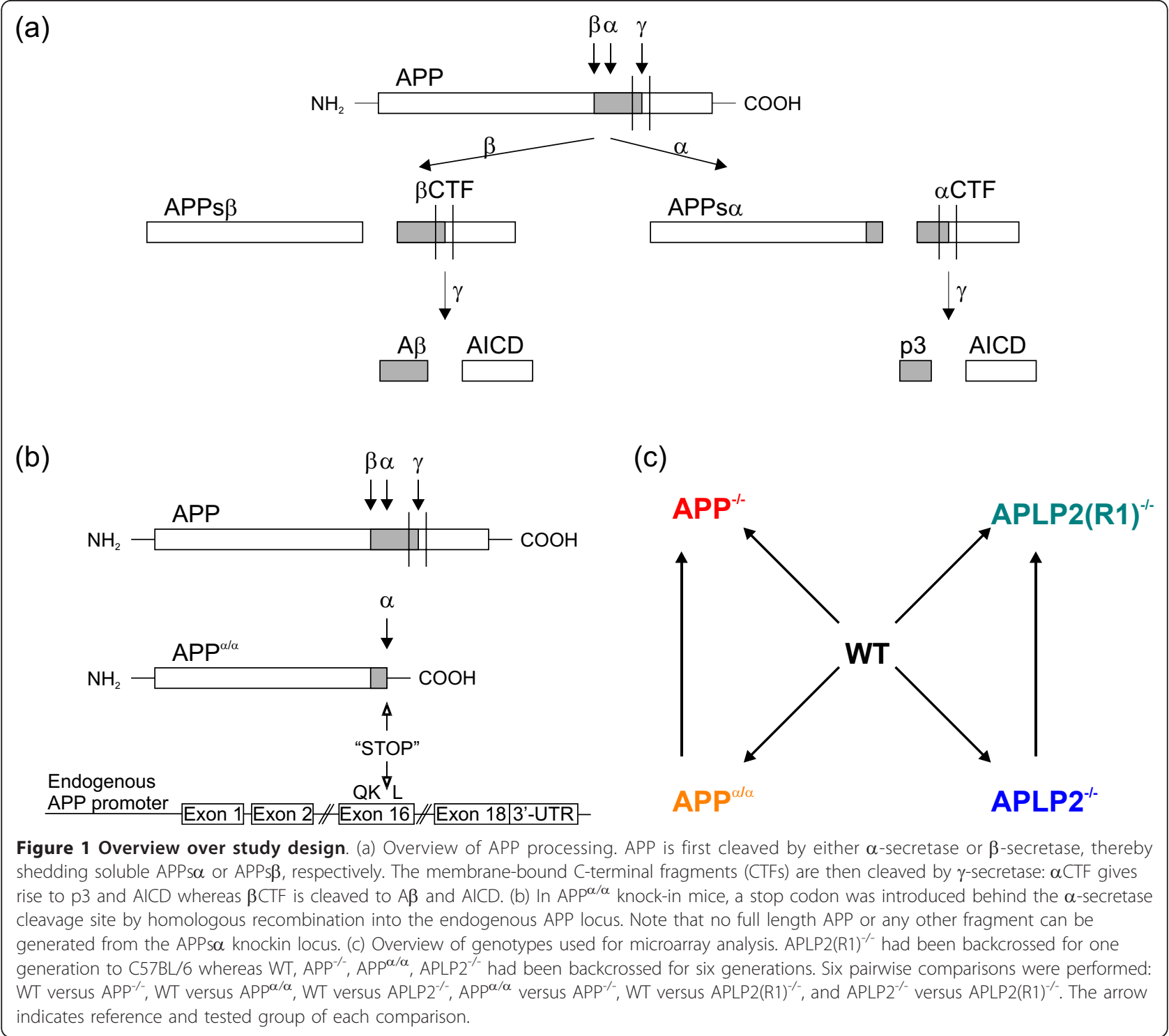


nucleus and can form a complex with the adaptor FE65 and the histone acetyltransferase TIP60. This complex can induce the transcription of artificial reporter constructs in transfected cells $[2,3]$. Likewise, APLP1 and APLP2 are subject to $\gamma$-secretase processing and can stimulate the expression of heterologous reporter constructs in an FE65-dependent manner [4]. Additional complexity comes from recent studies indicating that APP (and both APLPs) can form tripartite complexes with the adaptor protein MINT3 and the transcriptional co-activators TAZ and YAP. When overexpressed in HEK293 cells, this complex functions in GAL4 reporter assays [5,6]. To date, several putative AICD target genes have been identified (mainly using overexpression approaches) including Kai1 [7], Gsk3b [8,9], Nep [10], Egfr [11], p53 [12], Lrp [13], Tip60, Bace1, App itself [14] as well as genes involved in cytoskeletal dynamics [15]. However, the validity of these proposed targets, in particular regarding the question of whether they also constitute endogenous AICD/ALID target genes, has remained controversial [16-22]. Interestingly, in several recent studies, increased production of AICD either in transfected cells or in transgenic animals did not lead to a consistent up-regulation of previously proposed target genes [15,20,22].

Previously, we showed that knockout (KO) mice deficient in a single family member such as APP (or one of the APLPs) are viable $[23,24]$ whereas combined $\mathrm{APP}^{-/-}$ $\mathrm{APLP}^{-1-}$ or $\mathrm{APLP}^{-1-} \mathrm{APLP}^{-1-}$ double $\mathrm{KO}$ mice [24] and $\mathrm{APP}^{-1-} \mathrm{APLP}^{-1-} \mathrm{APLP}^{-1-}$ triple mutants [25] die shortly after birth, likely due to defects of neuromuscular transmission [26]. Neither $\mathrm{APP}^{-/-}$nor APLP2 ${ }^{-/-}$mice display obvious defects of central nervous system (CNS) morphology, yet $\mathrm{APP}^{-1-}$ mice revealed reduced body weight and defects in spatial learning associated with impaired synaptic plasticity including long-term potentiation (LTP) [26]. However, the molecular mechanisms underlying these defects have remained unclear.

Processing of APP gives rise to several fragments including besides neurotoxic $A \beta$ the $\alpha$-secretase-generated soluble APPs $\alpha$ fragment that is neuroprotective and involved in synaptic plasticity $[27,28]$. To delineate its specific functions, we previously generated APPs $\alpha$ knockin $\left(\mathrm{APP}^{\alpha / \alpha}\right)$ mice by inserting via gene targeting a stop codon into the endogenous APP locus right after the $\alpha$-secretase cleavage site [28]. Thus, APP $^{\alpha / \alpha}$ knockin mice express only secreted APPs $\alpha$ from the endogenous APP promoter (Figure 1b).

Here, we employed a rational unbiased approach and investigated transcriptional changes arising due to the lack of APP family members in the adult cortex of knockout mice to gain further insight into the physiological and signaling functions of APP family members. This includes transcriptome changes that may arise due to a lack of direct AICD/ALID-mediated transcriptional regulation as well as changes resulting from indirect signaling events mediated by transmembrane APP/APLP isoforms. First, we analyzed transcriptome changes due to the complete absence of APP or APLP2 (including all their proteolytic fragments) by conducting the pairwise comparisons of WT versus $\mathrm{APP}^{-/-}\left(\mathrm{WT} / \mathrm{APP}^{-/}\right)$and WT versus APLP2 $^{-/-}\left(\mathrm{WT}_{\mathrm{APLP2}}{ }^{-/}\right)$. Second, we had a closer look at the role of different APP fragments, in particular APPs $\alpha$. Therefore, we compared the transcriptome of $\mathrm{APP}^{\alpha / \alpha}$ mice both to WT (WT/APP ${ }^{\alpha / \alpha}$ ) and $\mathrm{APP}^{-/-}$ mice $\left(\mathrm{APP}^{\alpha / \alpha} / \mathrm{APP}^{-/-}\right)$, respectively. Third, we addressed the influence of the genetic background by comparing knockout animals of mixed $129 \times \mathrm{C} 57 \mathrm{BL} / 6$ genetic background (APLP2 $(\mathrm{R} 1)^{-/-}$) to those backcrossed to C57BL/ 6 for 6 generations.

\section{Results and Discussion}

We subjected prefrontal cortices of adult male mice (24 28 weeks of age) of the following groups to transcriptome analysis: WT $(\mathrm{n}=3), \mathrm{APP}^{-/-}(\mathrm{n}=3), \operatorname{APP}^{\alpha / \alpha}(\mathrm{n}=3)$, APLP2 $^{-1-}(n=3), \operatorname{APLP} 2(R 1)^{-1-}(n=3)$ (Figure 1c). WT, $\mathrm{APP}^{-/-}, \mathrm{APP}^{\alpha / \alpha}, \mathrm{APLP}^{-/-}$had been backcrossed for six generations to $\mathrm{C} 57 \mathrm{BL} / 6$ mice. APLP2(R1) $)^{-1-}$ mice harbor the identical knockout allele as APLP2 ${ }^{-/-}$but were only backcrossed once. Note that $\mathrm{APP}^{-/-}$mice lack membraneanchored full length APP (APP-FL) as well as all proteolytic fragments derived from it (APPs $\alpha, A \beta, A P P s \beta, \alpha C T F$, $\beta C T F$ and AICD), whereas APP ${ }^{\alpha / \alpha}$ mice express APPs $\alpha$ but lack full length APP and all other fragments.

Raw data was processed according to the RMA procedure $[29,30]$. We validated the microarray data by clustering the processed raw data based on all available App/ Aplp2 probe sets. As expected, all samples grouped according to their genotypes: WT, $\mathrm{APP}^{-/-}, \mathrm{APP}^{\alpha / \alpha}$, and APLP2 $2^{-/-}$ samples were clearly separated (Additional file 1).

\section{Differential gene expression in mice lacking APP family members}

First, we wanted to address the question of which impact each genotype has on transcription by searching for genes that show differential expression in the different comparisons. To identify significantly up- or downregulated genes, we performed a Significance Analysis of Microarrays (SAM) with a False Discovery Rate (FDR) of app. 5\% (see Table 1 and Additional file 2).

A total of 359 genes (274 up- and 85 down-regulated) were differentially expressed in $\mathrm{WT} / \mathrm{APP}^{-1-}$. The comparison WT/APLP2 ${ }^{-/}$led to 1242 differentially expressed genes (1142 up- and 100 down-regulated). For the comparison WT/APP ${ }^{\alpha / \alpha}$, we observed 447 significantly regulated genes (250 up- and 197 down-regulated). In contrast, we only observed 29 significant genes in the comparison $\mathrm{APP}^{\alpha / \alpha} / \mathrm{APP}^{-/-}$(all of them were upregulated). Based on the total number of significant 
Table 1 Overview over differentially expressed genes (R6 animals)

\begin{tabular}{|c|c|c|c|c|c|c|}
\hline & \multicolumn{2}{|c|}{ total } & \multicolumn{2}{|c|}{ up } & \multicolumn{2}{|c|}{ down } \\
\hline & $\mathrm{FC} \geq 2$ & no FCC & $\mathrm{FC} \geq 2$ & no FCC & $\mathrm{FC} \geq 2$ & no FCC \\
\hline$\overline{\text { WT/APP }}-/-$ & 11 & 359 & 2 & 274 & 9 & 85 \\
\hline WT/APLP2 ${ }^{-/-}$ & 11 & 1242 & 6 & 1142 & 5 & 100 \\
\hline$\overline{\mathrm{WT} / \mathrm{APP}} \alpha / \alpha$ & 15 & 447 & 2 & 250 & 13 & 197 \\
\hline $\mathrm{APP}^{\alpha / \alpha} / \mathrm{APP}^{-/-}$ & 2 & 29 & 2 & 29 & 0 & 0 \\
\hline
\end{tabular}

The number of significant genes is displayed for all relevant comparisons if at least one probe set identifier of the gene meets the respective criteria. Note that probe sets for App and Aplp2 are not part of the indicated numbers. Numbers indicate significant genes either with fold change criterion ( $F C \geq 2$, highlighted in bold) or without fold change criterion (no FCC). Note that all animals had been backcrossed for 6 generations to C57BL/6 (R6).

differentially expressed genes in these comparisons, the APLP2 knockout has the highest impact on gene expression.

To get an idea how many differentially expressed genes with high fold changes are within each list, we introduced a fold change criterion and determined the number of genes that differ by at least 2-fold (Table 1). In the comparisons $\mathrm{WT}_{\mathrm{APP}} \mathrm{AP}^{-/} 11$ genes, in $\mathrm{WT} /$ $\mathrm{APLP}^{-/-} 11$ genes, in WT/APP $\mathrm{W}^{\alpha / \alpha} 15$ genes, and in $\mathrm{APP}^{\alpha / \alpha} / \mathrm{APP}^{-/-} 2$ genes passed this criterion. This shows that the majority of significant differentially expressed genes show only small to moderate (up to 2-fold) alterations in gene expression. This is consistent with previous studies [31] and likely due to the complex nature of cortical tissue consisting of a multitude of neuronal and glial subpopulations. In $\mathrm{APP}^{-/-}$and $\mathrm{APP}^{\alpha / \alpha}$ animals, no compensatory up-regulation of Aplp1 and Aplp2 at the mRNA level was observed. Likewise, no up-regulation of $A p p$ and Aplp1 was observed in APLP2 ${ }^{-/-}$animals thus confirming previous Western blot results [24].

\section{Analysis of biological pathways affected in APP/APLP knockout mice}

Subsequently, we analyzed the list of significant genes from all comparisons using DAVID bioinformatics resources [32]. Within DAVID, we did Functional Annotation Clustering using Gene Ontology terms (biological processes) and pathway databases (Biocarta, Reactome, Panther, KEGG) to gain an overview about the nature of genes and potential shared functional pathways.

We found 24 enriched clusters in the comparison $\mathrm{WT} \mathrm{APP}^{-/-}, 29$ in WT/APP ${ }^{\alpha / \alpha}$, and 35 in WT/APLP2 $2^{-/-}$ (Additional file 3). Due to the low number of significant genes in $\mathrm{APP}^{\alpha / \alpha} / \mathrm{APP}^{-/-}$, no gene set enrichment could be assessed. Interestingly, several of these enriched clusters were shared between the different pairwise comparisons including regulation of neurogenesis, transcription and kinase activity (Figure 2, Additional file 3).
The finding that lack of either APP or APLP2 affects expression of genes involved in neurogenesis confirms and further extends previous studies that implicated APP in neuronal progenitor regulation [33-35]. In APPoverexpressing transgenic mouse models, adult neurogenesis in the hippocampus is impaired [36] which has been mainly attributed to $A \beta$-mediated toxic effects. Regulation of transcription was identified as another shared cluster between WT/APP ${ }^{-/-}$and WT/APLP2 $2^{-/-}$ pointing towards similar functions of APP family members in this cellular process, possibly via AICD/ALID signaling or via more indirect mechanisms. Shared functional clusters were also found for $\mathrm{WT} / \mathrm{APP}^{-/}$and WT/ $\mathrm{APP}^{\alpha / \alpha}$, namely neurogenesis and negative regulation of protein kinase activity which may indicate that phenotypes of APP-KO mice, e.g. defects in synaptic plasticity, arise due to alterations in the phosphorylation state of yet to be identified target proteins.

Although A $\beta$ serves as a central trigger for AD pathogenesis, the physiological role of APP and the question of whether a loss of its functions contributes to AD are still unclear. We therefore investigated a possible enrichment of genes previously linked to Alzheimer's disease in our dataset. Comparing the 359 genes differentially expressed in WT/ $\mathrm{APP}^{-/-}$with the AlzGene dataset (currently comprising 662 genes), we identified 14 genes, namely Abcg4, Ache, Aldh2, Arsb, Bcl2, Bdnf, Crh, Egr2, Fos, Gstz1, Hspa1a, Hspa1b, Hspa5, Ppp1r3c. Next, we assessed whether this number of 14 identified genes represents a significant enrichment of AD-related genes in the WT/ $\mathrm{APP}^{-/}$dataset. To this end, we randomly drew 100 gene sets of the same size $(n=359)$ from the pool of genes covered by the array and checked them against the AlzGene set. We found an average of 9 genes per randomly drawn gene set and used this as reference for Fisher's exact test. However, no significant enrichment of genes from the AlzGene dataset was present in $\mathrm{WT} / \mathrm{APP}^{-/}$.

\section{Proposed AICD target genes show only a minor or no significant differential expression in APP- and APLP2- deficient cortex}

Several genes have been proposed to be directly regulated at the transcriptional/promoter level by an AICD/ FE65 transcriptionally active complex including Bace1 [14], Kai1 [7], Egfr [11], Gsk3b [8,9], p53 [12], Tip60 [14], and Vglut2 [37]. By array analysis we detected in $\mathrm{APLP}^{-/-}$mice for Vglut2 and Gsk3b a small yet significant up-regulation of 1.27 -fold and 1.2-fold, respectively, compared to wild-type animals (Additional File 2). In all other genotypes, including $\mathrm{APP}^{-/-}$, expression was, however, not significantly altered. To further validate these results we conducted a qPCR analysis of these proposed target genes (Figure 3). This way, we detected a 1.6-fold 


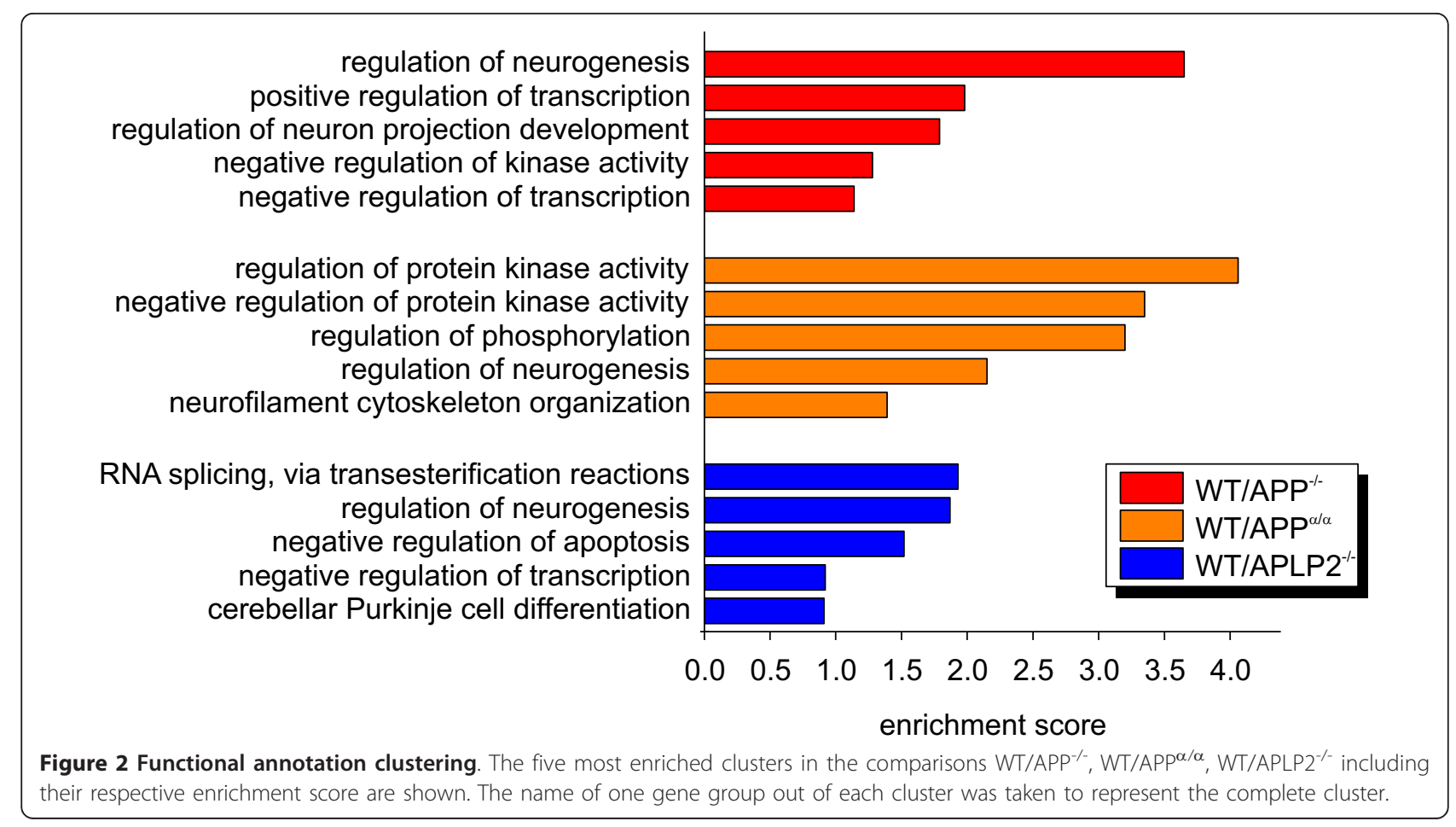

increase of Egfr mRNA expression solely in APLP2 $2^{-/-}$ animals, compared to wild type controls $(\mathrm{p}<0.05)$. Of note, qPCR analysis of $\mathrm{APP}^{-/-}, \mathrm{APP}^{\alpha / \alpha}$ and $\mathrm{APLP} 2^{-/-}$cortex failed to detect significant expression differences of all other tested candidate genes, including Vglut 2 and Gsk3b.
What might be the reasons that proposed target genes have proven difficult to confirm in follow-up studies including work reported here? A major reason may be the difference in experimental systems used as overexpression in cell lines may not necessarily reflect a role of AICD for endogenous gene expression. In line with

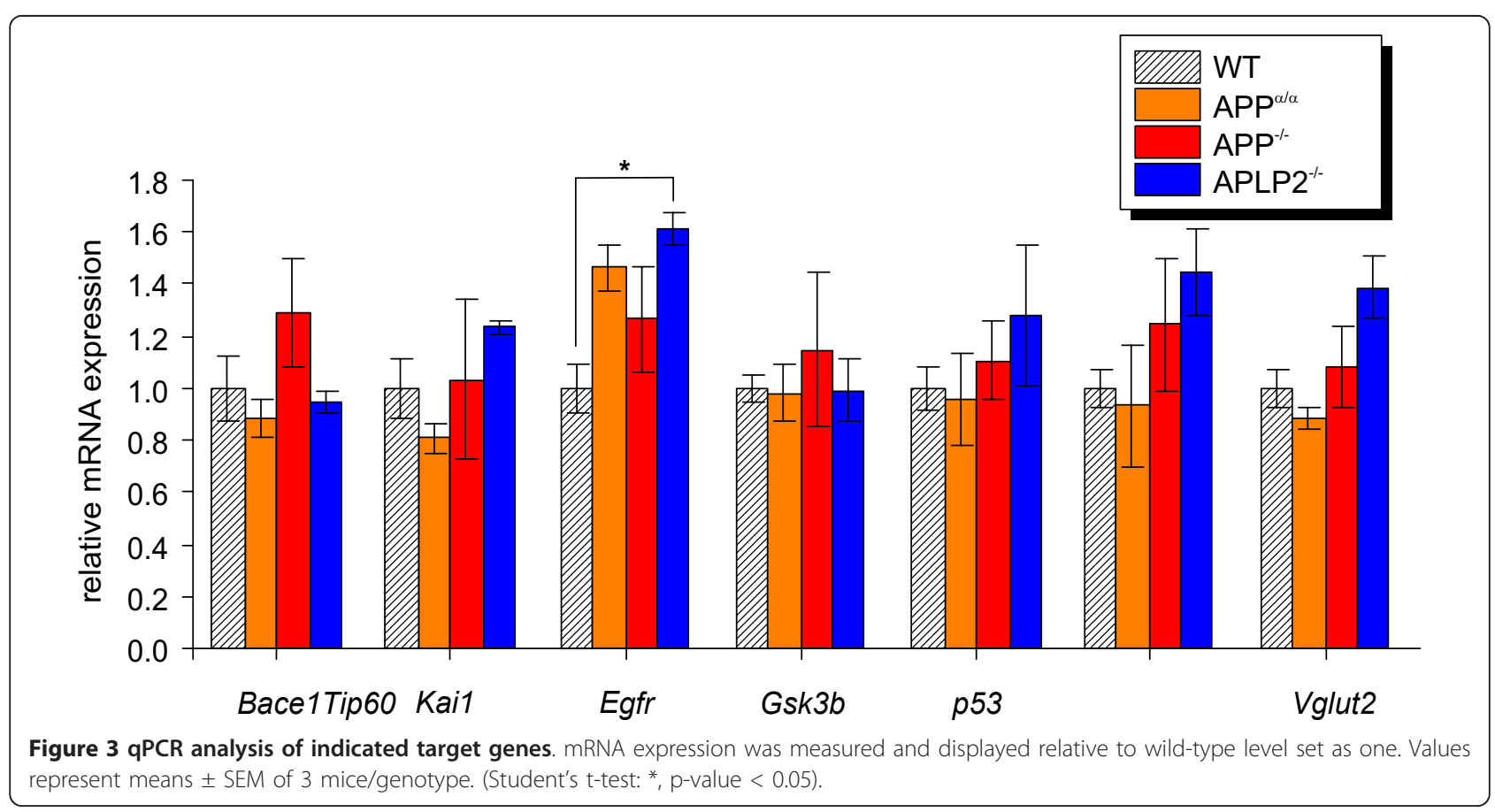


this study, we previously found no impact on Kai1, Gsk3a, Gsk3b, App , and Nep mRNA expression when treating different cell lines with the $\gamma$-secretase inhibitor DAPT or when assessing endogenous gene expression in AICD-deficient model systems [16]. In addition, as shown by the same study, clonal variability of immortalized fibroblast lines may lead to variable gene expression irrespective of either $\mathrm{APP}^{-/-}$or $\mathrm{APP}^{+/+}$ genotype [16]. On the other hand, one might expect an inverse regulation of target genes upon AICD deficiency as opposed to overexpression. A genome-wide microarray-based approach to detect AICD target genes used an inducible FE65/AICD cell line [15]. Here, no change in Kai1 and Gsk3b mRNA expression was detected. Similarly, Waldron et al. [22] found no alteration in mRNA expression of Kai1, Bace1, Egfr, Tip60, and p53 in AICD-enriched FE65-tranfected cells. Moreover, transcriptome analysis in AICD transgenic mouse brain revealed no apparent difference between transgenic animals and littermate controls [20] and qPCR analysis of proposed target genes, including those studied here, failed to detect significant changes in mRNA expression. Overall, our results are highly consistent with these studies. Although our study clearly indicates that AICD or ALID2 are on their own not essential transcriptional regulators of tested target genes in adult prefrontal cortex, we cannot exclude at present that other APP family members (including APLP1) may at least partially compensate for a single gene deficiency. Due to the lethality of combined mutants shortly after birth we had previously analyzed the expression of a subset of target genes in $\mathrm{APP}^{-/} \mathrm{APLP}^{-/-}$embryonic brain and fibroblasts [16]. As APLP1 is not expressed in fibroblasts, $\mathrm{APP}^{-1-} \mathrm{APLP}^{-1-}$ fibroblasts (compared to APP retransfected cells) provide a cellular model in which all APP family members are lacking. However, neither Nep nor $G s k 3 b$ expression was significantly affected in either embryonic brain or fibroblasts [16]. A global assessment of transcriptome changes in adult brain lacking multiple APP family members (a tissue more relevant for AD) will await the generation of viable conditional mutants. Considering the complexity of cortical tissue, it is still possible that gene expression differences occurring only in distinct cell types may remain below the detection limit of our analysis. In line with this hypothesis, Schrenk-Siemens et al [37] reported a reduction of Vglut2 mRNA and VGLUT2 protein expression in glutamatergic neurons obtained by retinoic acid differentiation of $\mathrm{APP}^{-/-} \mathrm{APLP}^{-/-}$embryonic stem cells whereas in this study no difference was detectable in cortical tissue. It remains to be seen whether regulation of other target genes might also be cell type-specific.

\section{Genes co-regulated due to the lack of either APP or APLP2}

As there is genetic $[24,25]$ and cell biological evidence (such as a shared set of protein interaction partners [38]) that APP family members serve related physiological functions, we searched for genes that are, compared to wild-type, differentially regulated in more than one genotype (Figure 4a). To this end, we created a Venn diagram of the pairwise comparisons $\mathrm{WT} / \mathrm{APP}^{-1-}$ and WT/APLP2 ${ }^{-1-}$. We found 213 probe set identifiers (Additional file 4) representing 181 known genes that are differentially expressed in both cases (Figure 4b) and regulated in the same direction as shown by cluster analysis (Figure 4c). Furthermore, we analyzed how many of these genes were in addition significantly regulated in WT/APP ${ }^{\alpha / \alpha}$. Out of 181 genes co-regulated by lack of either APP or APLP2, 97 were also found in the comparison WT/APP ${ }^{\alpha / \alpha}$ and regulated in the same direction (Figure 4d, Additional file 4). Thus, functional similarities of APP family members are also reflected at the transcriptional level by co-regulated gene sets in the respective loss-of-function mutants. To investigate this more closely we further examined two of these genes by qPCR analysis: heat shock protein 5 (Hspa5) and cyclindependent kinase inhibitor 1A (Cdkn1a).

Hspa 5 attracted our attention for two reasons. Amongst co-regulated genes we found a consistent down-regulation of four heat-shock proteins including besides Hspa5, Hspa1b, Hspb1, and Hsph1. HSPA5 (also known as GRP78) is an ER chaperone involved in the ER stress response and had previously been shown to interact with APP and modulate A $\beta$ production [39]. In addition, GRP78 was recently identified as a gene that may counteract the proliferative effect of secreted APPs in tumor models [40]. As functional annotation clustering identified neurogenesis as a pathway affected in all genotypes compared to wild-type, it was interesting to find $C d k n 1 a$, also known as $p 21$, amongst these co-regulated genes. Based on array analysis, both $C d k n 1 a$ and Hspa 5 were down-regulated by about two-fold in all three mutant genotypes compared to WT (Figure 5a). Employing qPCR we confirmed these results and found again a significant down-regulation of about the same magnitude (Figure $5 b$ ).

It is noteworthy that in APP-overexpressing transgenic mice Hspa 5 had previously been found to be up-regulated [41] suggesting an inverse transcriptional regulation as a consequence of either loss or gain of APPdependent signaling. The CDK inhibitor p21 has been shown to restrict adult neurogenesis in the hippocampus, as evidenced by increased proliferation of neuronal progenitors in $\mathrm{p} 21^{-1-}$ mice [42]. Given the Cdkn1a/p21 down-regulation we found here, one might thus expect $\mathrm{APP}^{-1-}$ (or $\mathrm{APLP}^{-/-}$) mice to show dysregulated 
(a)

\section{$\mathrm{APP}^{-1-}$}

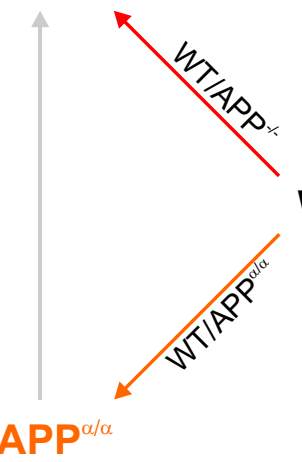

APLP2(R1)

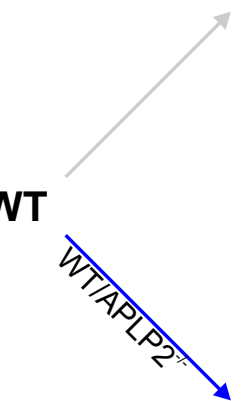

(c)

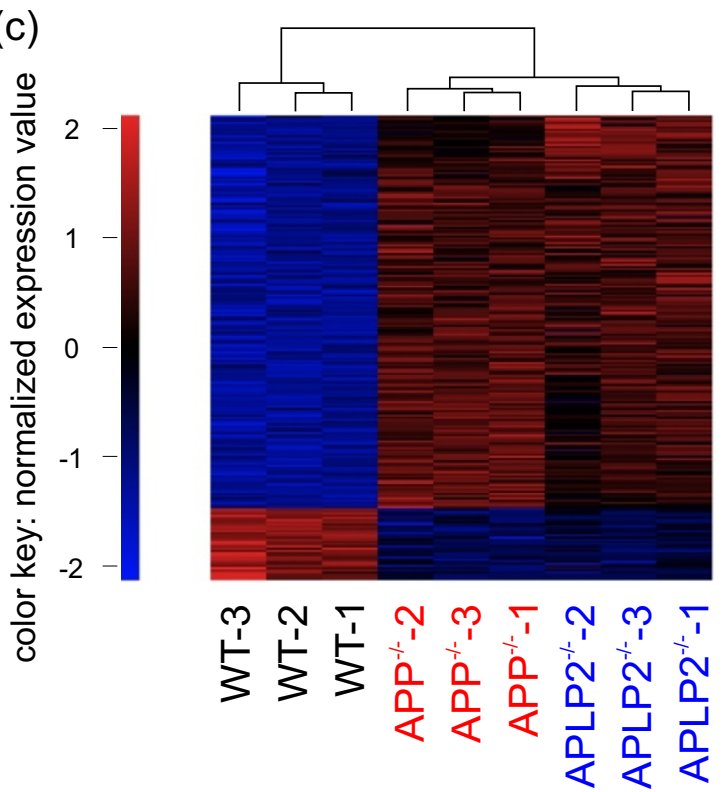

(b)

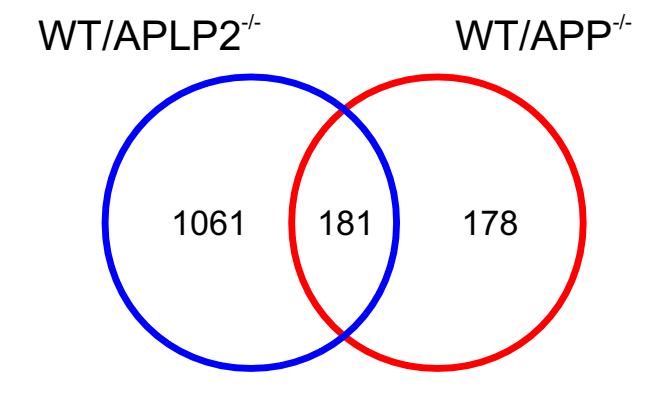

APLP2 $2^{-/-}$ (d)

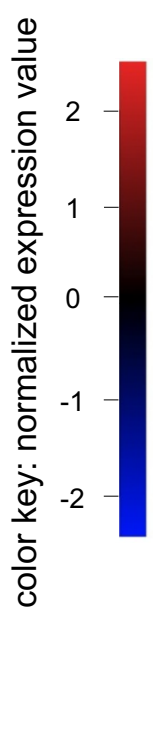

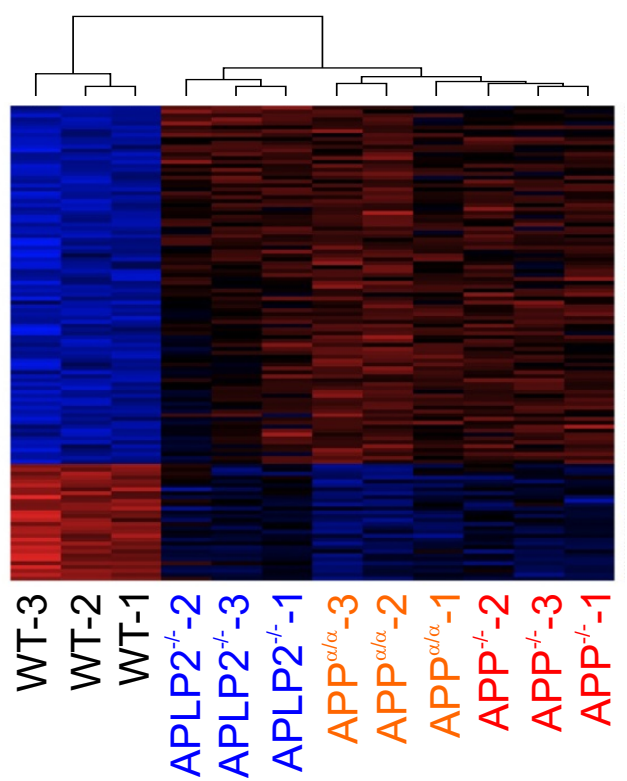

Figure 4 Co-regulation of genes in WT/APP ${ }^{-/-}$and WT/APLP2 ${ }^{-/-}$. (a) Overview of the analyzed comparisons. (b) Venn diagram of the two comparisons WT/APP ${ }^{-/}$and WT/APLP2 ${ }^{-/-}$based on gene lists obtained by significance analysis. The number of significant genes is indicated in the respective segments. Note that 181 genes are co-regulated by the lack of either APP or APLP2. (c) Heatmap of the 213 identifiers corresponding to the set of 181 genes that are co-regulated in both pairwise comparisons. All identifiers show differential expression in the same direction. (d) Heatmap of 122 identifiers corresponding to the set of 97 genes that are found in both pairwise comparisons as well as in WT/APP ${ }^{\alpha / \alpha}$. The values of the heatmaps $(c, d)$ are normalized expression values with red and blue color representing the number of standard standard deviations above or below the mean expression for each probe set, respectively.

neurogenesis. On the other hand, we had previously shown that endogenous APPs and APLP2s play a crucial role as growth factors for neuronal stem cells in the adult subventricular zone (SVZ) [35]. Depletion of APPs $\alpha$ by infusion of APP-binding antibodies or as a consequence of pharmacological inhibition of APPs $\alpha$ production reduced the number of neuronal progenitor cells in the SVZ [35]. Thus neurogenesis might be under complex control of APP-mediated signaling pathways, both by membrane-anchored APP and secreted APP isoforms.
Role of APP domains for transcriptome changes

Next, we compared the transcriptome of $\mathrm{APP}^{-/-}$mice to that of $\mathrm{APP}^{\alpha / \alpha}$ animals (Figure 6a). Specifically, we wanted to answer the question of whether the transcriptome of $\mathrm{APP}^{\alpha / \alpha}$ mice would be more similar to that of WT mice (which would indicate that APP-FL and/or the APP C-terminus is of minor importance), or would rather resemble that of $\mathrm{APP}^{-/-}$cortices (which would indicate an important role of APP-FL and/or APP Cterminal fragments for signaling). As APPs $\alpha$ is sufficient to rescue the learning impairment and LTP defect of 


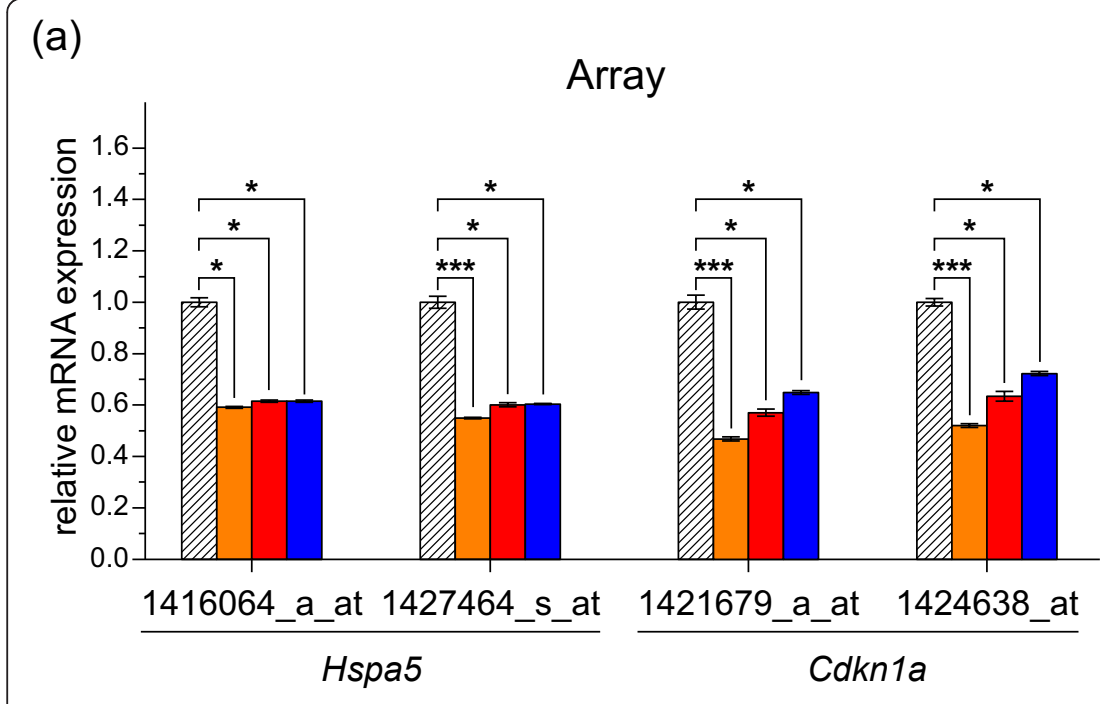

Figure 5 Relative mRNA expression of selected genes co-regulated in all comparisons. (a) Relative mRNA expression obtained by array analysis. Values of indicated probe set identifiers were compared to WT levels. Values represent relative expression value \pm relative standard deviation. Differences were tested for significance by SAM (*, q-value $<0.05 ;{ }^{* *}$, q-value $<0.01$; ***, q-value $\left.<0.001\right)$. (b) The corresponding mRNA expression was measured by GPCR and displayed relative to wild-type level set as one. Values represent means \pm SEM of 3 animals per group. (Student's t-test: ${ }^{*}$, p-value $<0.05 i^{* *}, \mathrm{p}$-value $<0.01$; ${ }^{* *}$, $\mathrm{p}$-value $<0.001$ ).

$\mathrm{APP}^{-/-}$mice [28], we initially inferred that this would also apply for the transcriptome of $\mathrm{APP}^{\alpha / \alpha}$ mice. However, in the significance analysis, $\mathrm{APP}^{\alpha / \alpha} / \mathrm{APP}^{-/-}$was the comparison with the lowest number of significant genes whereas the number of significant differentially expressed genes for $\mathrm{WT} / \mathrm{APP}^{-/-}$and $\mathrm{WT} / \mathrm{APP}^{\alpha / \alpha}$ was at least 10 times higher (see Table 1) indicating a close resemblance of $\mathrm{APP}^{\alpha / \alpha}$ and $\mathrm{APP}^{-/-}$samples. Next, we generated a Venn diagram to gain an overview on the absolute number of significant genes found in each intersection (Figure 6b, Additional file 5). A high proportion of genes significant in $\mathrm{WT} / \mathrm{APP}^{\alpha / \alpha}$ was also significant in $\mathrm{WT} / \mathrm{APP}^{-/-}$leading to a subset of 169 co-regulated genes. Second, to get a more quantitative understanding on this part of the dataset, we calculated the percentage of probe sets that were co-regulated between the different pairwise comparisons. To this end, we ranked all probe sets according to their absolute significance score and used the most significant 200 probe sets of each of these pairwise comparisons to generate the Venn diagram (Figure 6c). WT/APP ${ }^{-/}$and $\mathrm{WT} / \mathrm{APP}^{\alpha / \alpha}$ have $40 \%$ of the 200 probe sets in common whereas $\mathrm{WT} / \mathrm{APP}^{-/-}$and $\mathrm{APP}^{\alpha / \alpha} / \mathrm{APP}^{-/-}$share only 7 out of 200 probe sets (Figure 6c). Taken together these results suggest that with regard to transcriptional changes $\mathrm{APP}^{\alpha / \alpha}$ is functionally very similar to a complete APP knockout. These data may indicate an important role of full length APP and/or APP C-terminal fragments for direct or indirect signaling resulting in transcriptome changes. (b)
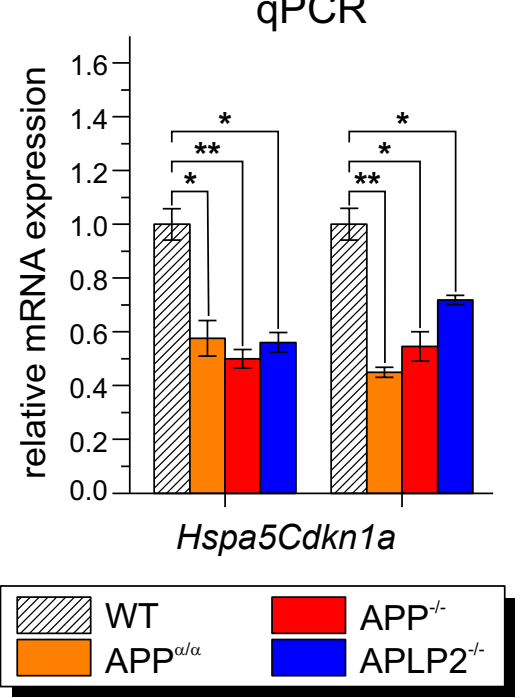

In addition, we raised the question whether the constitutive expression of APPs $\alpha$ would lead to changes in gene expression, as recently reported for APPs $\beta$ [43]. We found a small percentage $(8.5 \%)$ of probe sets in the intersection of $\mathrm{APP}^{\alpha / \alpha} / \mathrm{APP}^{-/-}$and WT/APP ${ }^{\alpha / \alpha}$ (Figure 6c). To investigate this finding further, we analyzed the 6 significant genes that are found within this group (Figure 6b) and used their corresponding 8 probe sets for a cluster analysis (Figure 6d). Hierarchical clustering results in a clear separation of $\mathrm{APP}^{\alpha / \alpha}$ from WT and $\mathrm{APP}^{-/-}$cortices. This points to a small subpopulation of genes that are actually regulated by the constant production of APPs $\alpha$ in the absence of APP full length and all other fragments.

Next, we took a closer look at genes co-regulated by the complete absence of APP or APP-FL as identified in the intersection of $\mathrm{WT} / \mathrm{APP}^{-/-}$and $\mathrm{WT} / \mathrm{APP}^{\alpha / \alpha}$ (Figure 6b). Interestingly, this gene set comprises several synaptic plasticity-related genes including the immediate early response factors Arc (activity-regulated cytoskeletonassociated protein), Fos (FBJ osteosarcoma oncogene), Egr2 (early growth response 2), and Dio2 (deiodinase, iodothyronine, type II), a key enzyme in the biosynthesis of the nuclear hormone Triiodothyronine (T3). Validation of these genes by qPCR (Figure 7) consistently identified an about 1.5- to 2-fold down-regulation in all genotypes and thus confirmed gene expression changes identified by array analysis. In case of Dio2, down-regulation in $\mathrm{APLP} 2^{-/-}$now reached significance level when using qPCR analysis as compared to array values. 


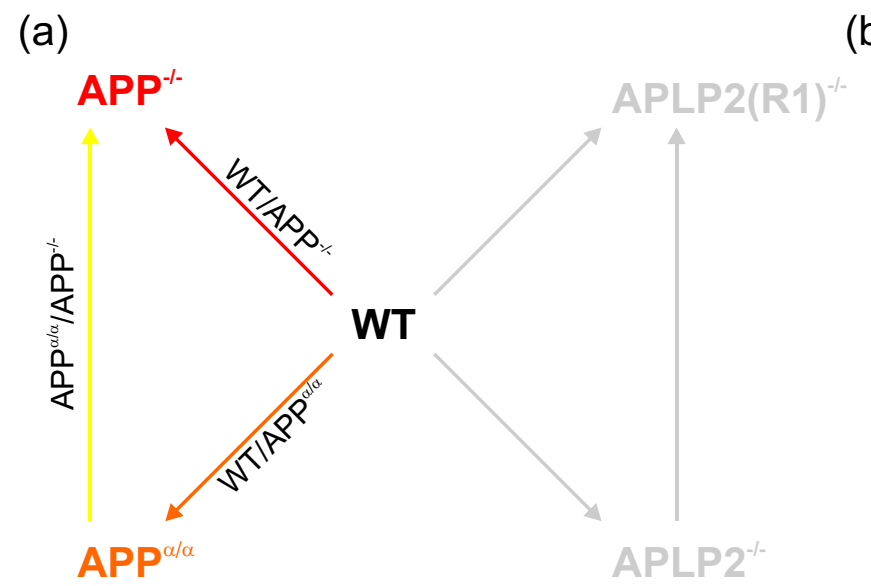

(b)

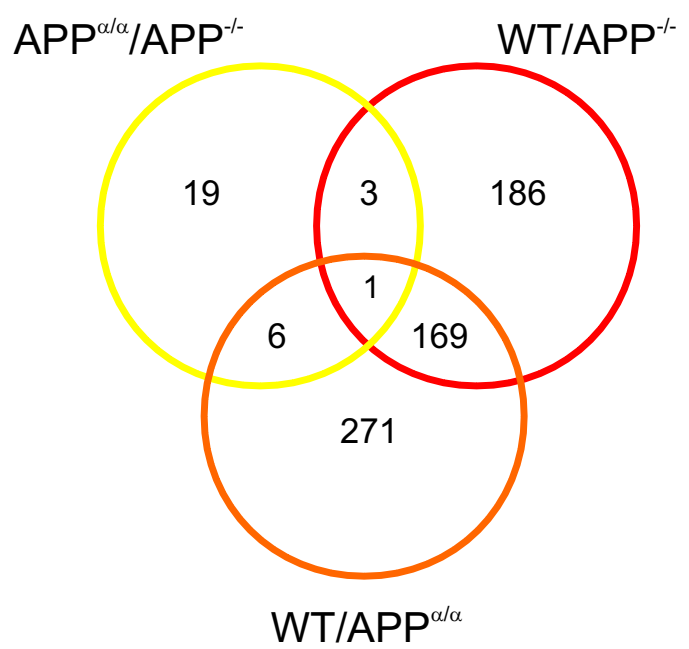

(c)

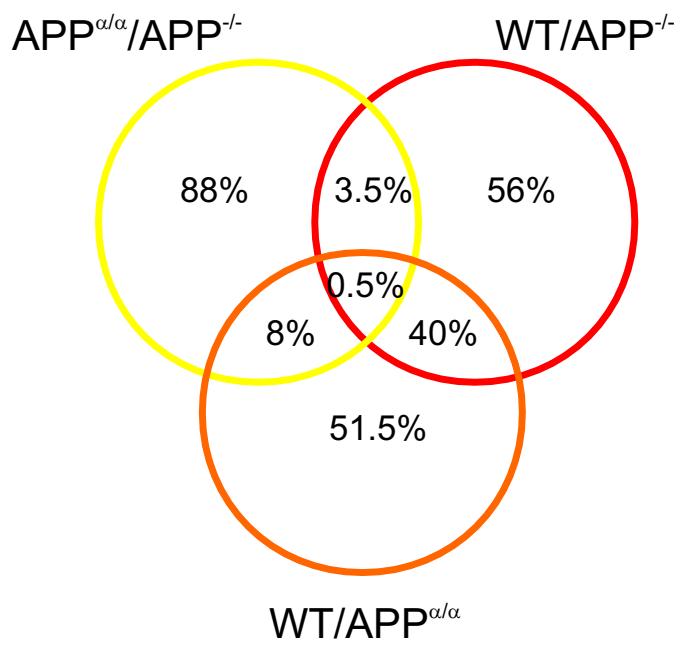

(d)

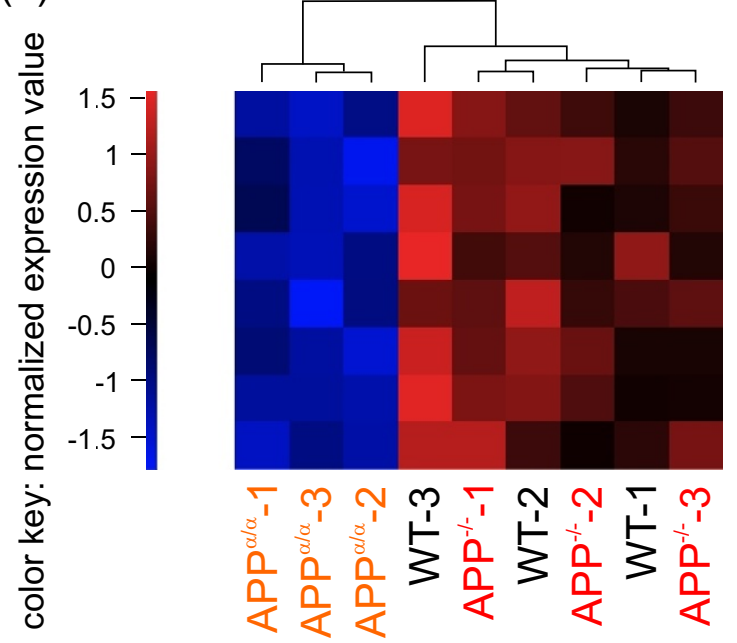

Figure 6 Analysis of common genetic profiles in WT/APP ${ }^{-/-}$, WT/APP ${ }^{\alpha / \alpha}$ and APP A $^{\alpha / \alpha} /$ APP $^{-/-}$. (a) Overview of the analyzed comparisons. (b) Venn diagram with SAM-based gene lists of the three comparisons WT/APP ${ }^{-/}$, WT/APP ${ }^{\alpha / \alpha}$ and APP ${ }^{\alpha / \alpha} /$ APP $^{-/-}$. The numbers indicate the absolute number of differentially expressed genes in the respective comparison. (c) Percentage of probe set identifiers co-regulated in two or three pairwise comparisons. Values are based on the 200 most significantly differentially expressed identifiers for each pairwise comparison. Note that $40 \%$ of probe sets are found in the overlap between WT/APP ${ }^{-/}$and WT/APP $\alpha / \alpha$. (d) Heatmap of the probe set identifiers corresponding to the set of 6 genes (b) in the intersection of WT/APP ${ }^{\alpha / \alpha}, \mathrm{APP}^{\alpha / \alpha} / \mathrm{APP}^{-/}$. The values of the heatmap are normalized expression values with red and blue color representing the number of standard standard deviations above or below the mean expression for each probe set, respectively.

Arc mRNA accumulates in activated synapses, modulates AMPAR trafficking and is critically involved in memory consolidation and LTP [44]. Both FOS, best known for its binding to the Jun/AP-1 transcription factor complex, and the $\mathrm{Zn}^{2+}$-finger transcription factor EGR2/KROX-20 are induced during neuronal activity $[45,46]$ and play an important role in learning and memory as well as LTP [46-48]. As $\mathrm{APP}^{-/-}$mice show an age-dependent deficit in spatial learning associated with impaired long-term potentiation (LTP), it was intriguing that we found a down-regulation of genes previously implicated in synaptic plasticity although further studies are needed to establish a causal link.

Influence of genetic background on gene transcription Genetic background is known to profoundly influence the occurrence, penetrance and severity of transgenic and knockout phenotypes, e.g. with regard to behavior or $\mathrm{A} \beta$ deposition $[49,50]$. For one of our mutants, APLP2 ${ }^{-1-}$, we had kept animals that had been backcrossed only once to C57BL/6 (designated APLP2(R1) ${ }^{-/-}$), whereas all other mutants had been backcrossed for six generations 


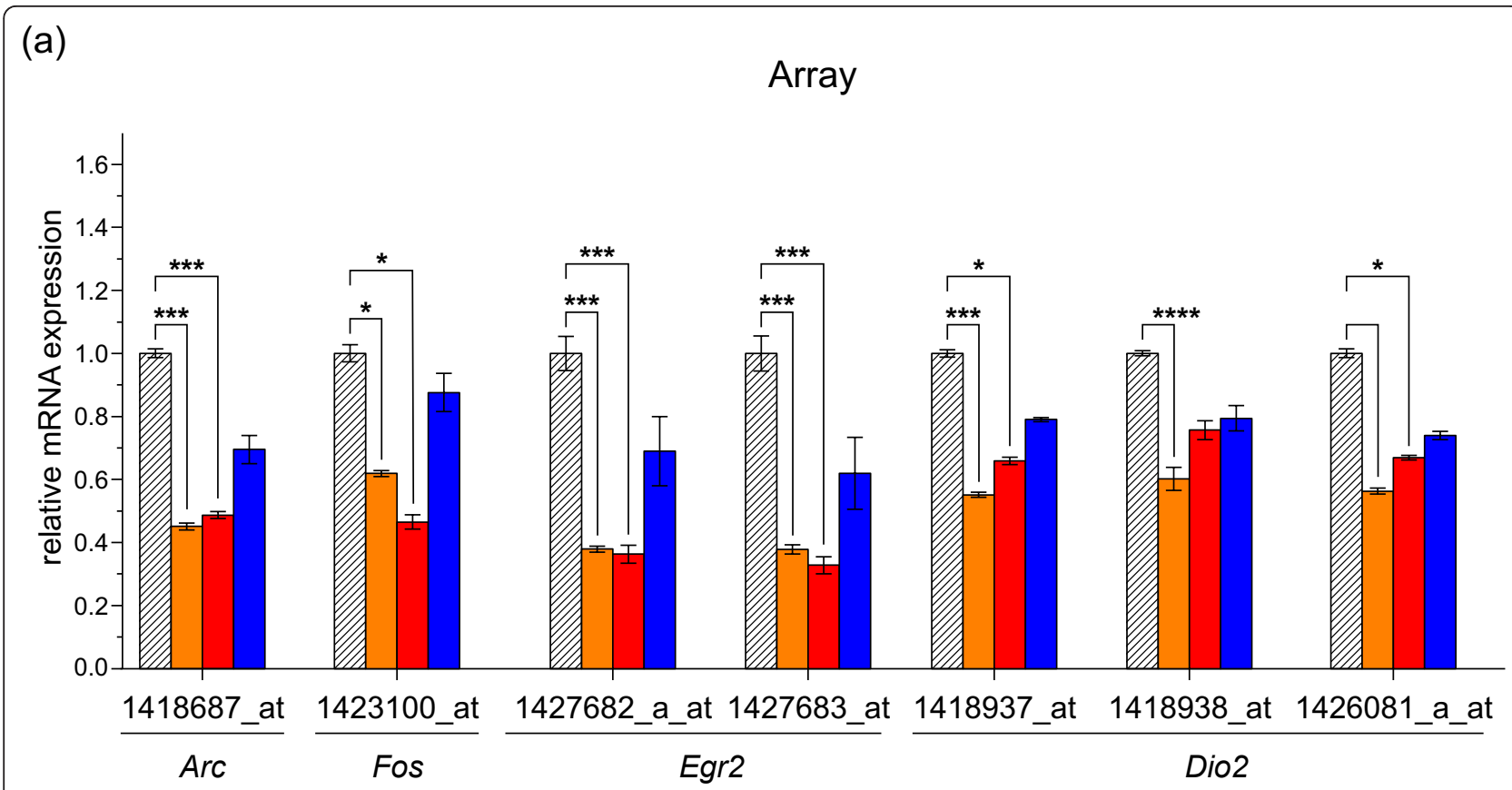

(b)
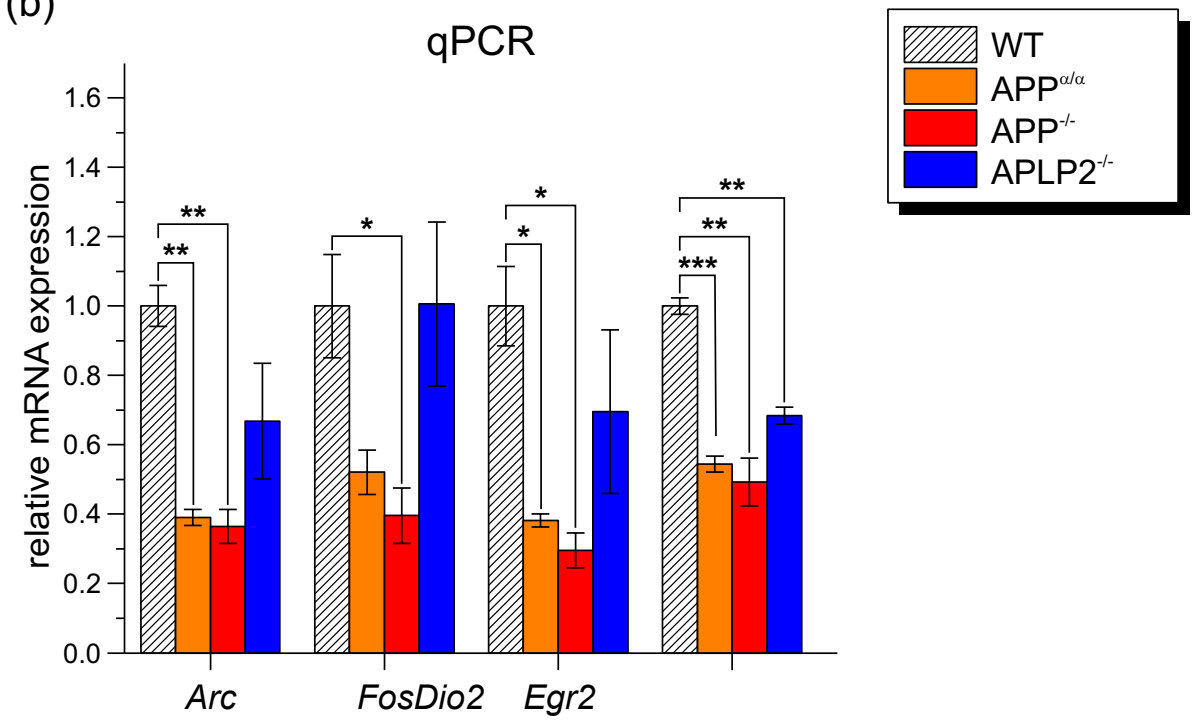

Figure 7 Relative mRNA expression of selected genes co-regulated in WT/APP ${ }^{-/-}$and WT/APP ${ }^{\alpha / \alpha}$. (a) Relative mRNA expression level obtained by array analysis. Values of indicated probe set identifiers/genes were compared to WT levels. Values represent relative expression value \pm relative standard deviation. Differences were tested for significance by SAM (*, q-value $<0.05$; ${ }^{* *}, \mathrm{q}^{*}$ value $\left.<0.01 ;{ }^{* * *}, \mathrm{q}^{*} \mathrm{value}<0.001\right)$. (b) The corresponding mRNA expression was measured by qPCR and displayed relative to wild-type level set as one. Values represent means \pm SEM of 3 animals per group. (Student's t-test: *, p-value $<0.05$; ${ }^{* *}, \mathrm{p}$-value $<0.01$; ${ }^{* *}$, p-value $<0.001$ ).

(designated R6). This allowed us to investigate the impact of genetic background on transcriptome changes versus changes that arise as a consequence of APLP2 gene deficiency (Figure 8a). To make this more clear for the reader, we will for the remaining study use the designation R6 and R1 for all subsequent comparisons (please note however that in figures 1234567 all animals were also of R6 genetic background).
The comparison APLP2(R6) $)^{-/-} / \operatorname{APLP} 2(\mathrm{R} 1)^{-/-}$, i.e. between animals of the same genotype but with different genetic background, led to 144 significant differentially expressed genes of which 50 were up- and 94 were down-regulated (Table 2, Additional file 6). For the comparison WT(R6)/APLP2(R1) $)^{-/-}$(animals with both different genotype and different genetic background) we found a total of 242 genes, 157 up-, 85 down-regulated 
(a)

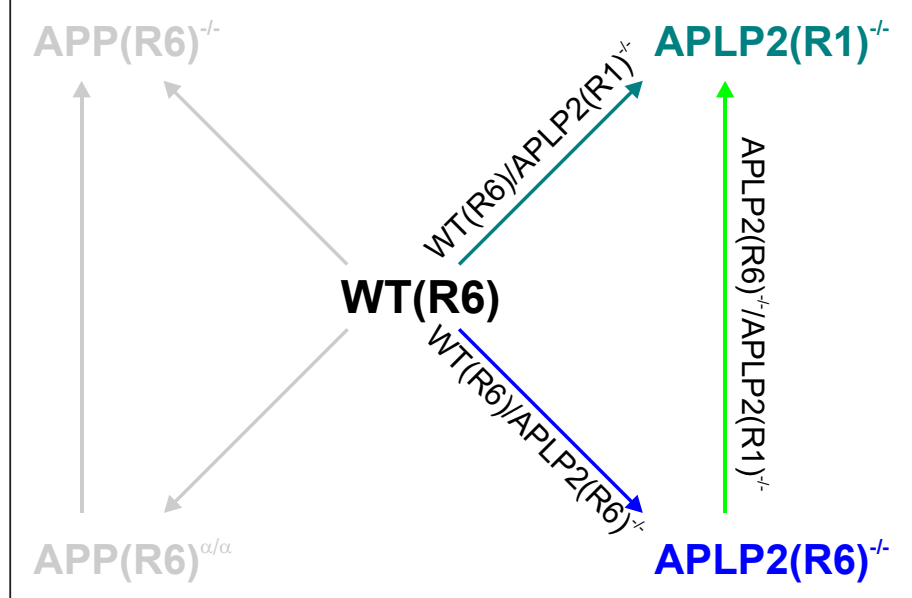

(b)

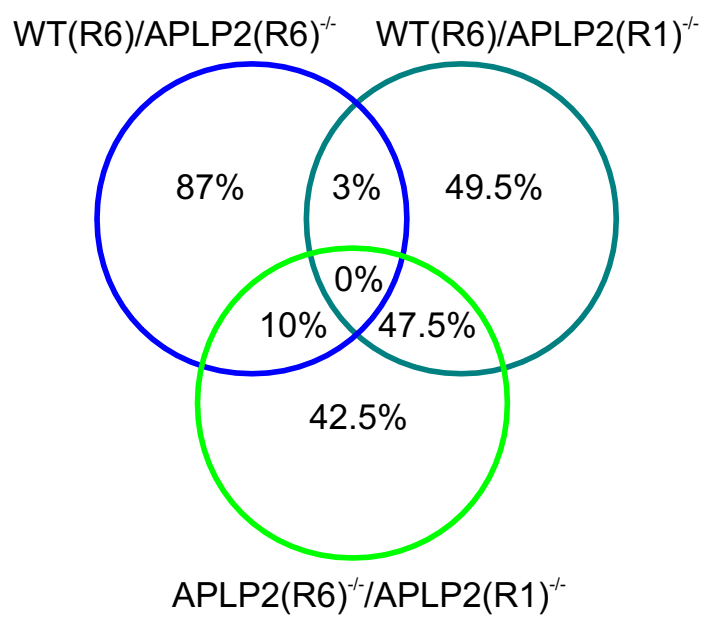

(c)

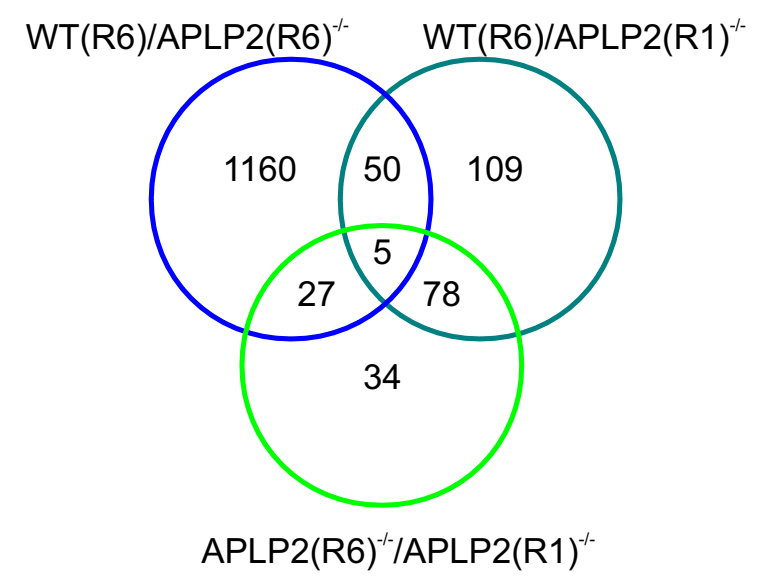

(d)

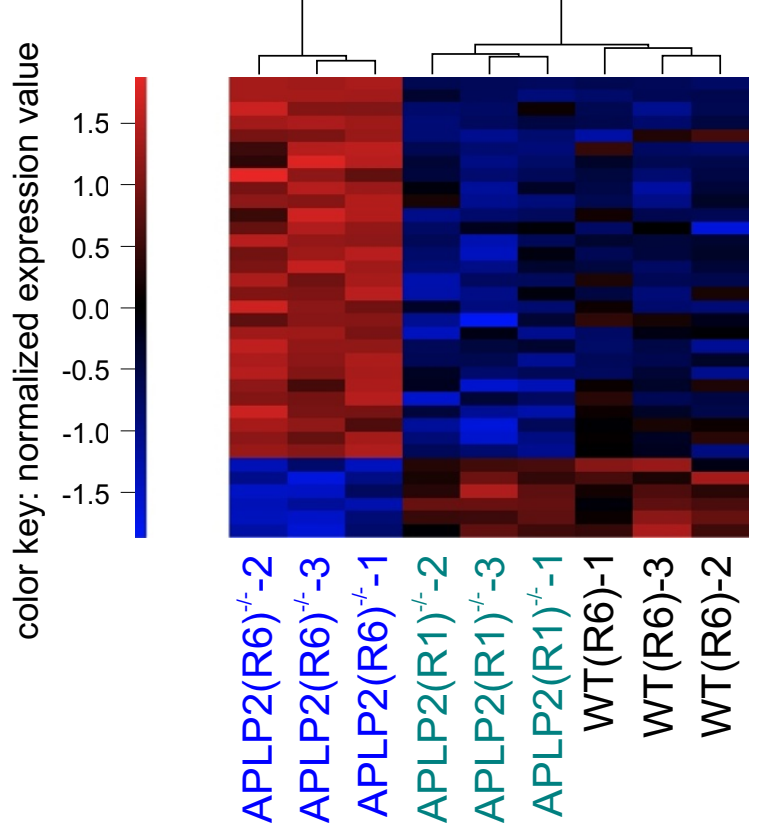

Figure 8 Co-regulation of genes in WT(R6)/APLP2(R6) $)^{-/-}$, WT(R6)/APLP2(R1) ${ }^{-/-}$, APLP2(R6) ${ }^{-/-} /$APLP2(R1) $)^{-/-}$. (a) Overview of the analyzed comparisons. (b) Percentage of probe set identifiers co-regulated in two or three pairwise comparisons. Values are based on the 200 most significantly differentially expressed identifiers for each pairwise comparison. (c) Venn diagram with SAM-based gene lists of the three comparisons WT(R6)/APLP2(R6) ${ }^{-1}$, WT(R6)/APLP2(R1) $)^{-/-}$and APLP2(R6) $)^{-/} / A P L P 2(R 1)^{-1}$. (d) Cluster analysis of probe sets corresponding to the set of 27 genes from (c) found exclusively in the comparisons WT(R6)/APLP2(R6) ${ }^{-1-}$ and APLP2(R6) $)^{-1 /} / A P L P 2(R 1)^{-1-}$. The values of the heatmap are normalized expression values with red and blue color representing the number of standard standard deviations above or below the mean expression for each probe set, respectively.

(Table 2, Additional file 6). The sizes of these two sets are more than 5 -fold smaller than the 1242 genes found for WT(R6)/APLP2(R6) ${ }^{-1-}$ (Table 2, Additional file 6). However, genetic background-related probe sets differentially

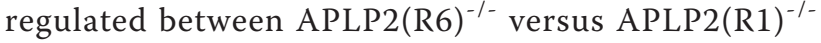
have very high significance scores due to high gene expression changes (Additional file 6). This considerable influence of genetic background is best reflected in the corresponding volcano plot (Figure 9) yielding, when compared to WT(R6)/APLP2(R6) ${ }^{-1-}$ (Figure 9b), a much larger number of probe sets with both high significance score (arbitrarily set to 6) and high fold change in the comparison APLP2(R6) $)^{-/-} /$APLP2(R1) ${ }^{-1-}$ (Figure 9a). Of note, only three probe sets matching these criteria were 
Table 2 Overview over differentially expressed genes: impact of genetic background

\begin{tabular}{|c|c|c|c|c|c|c|}
\hline & \multicolumn{2}{|c|}{ total } & \multicolumn{2}{|c|}{ up } & \multicolumn{2}{|c|}{ down } \\
\hline & $\mathrm{FC} \geq 2$ & $\begin{array}{l}\text { no } \\
\text { FCC }\end{array}$ & $\mathrm{FC} \geq 2$ & $\begin{array}{l}\text { no } \\
\text { FCC }\end{array}$ & $\mathrm{FC} \geq 2$ & $\begin{array}{l}\text { no } \\
\text { FCC }\end{array}$ \\
\hline$\overline{\mathrm{WT}(\mathrm{R} 6) / \mathrm{APLP} 2(\mathrm{R} 6)^{-/-}}$ & 11 & 1242 & 6 & 1142 & 5 & 100 \\
\hline$\overline{\mathrm{WT}(\mathrm{R} 6) / \mathrm{APLP} 2(\mathrm{R} 1)^{--}}$ & 35 & 242 & 19 & 157 & 16 & 85 \\
\hline $\begin{array}{l}\text { APLP2(R6) } \\
(\mathrm{R} 1)^{-/-} / \mathrm{APLP} 2\end{array}$ & 40 & 144 & 19 & 50 & 21 & 94 \\
\hline
\end{tabular}

The number of significant genes is displayed for all relevant comparisons if at least one probe set identifier of the gene meets the respective criteria. Note that probe sets for App and Aplp2 are not part of the indicated numbers. Numbers indicate significant genes either with fold change criterion ( $F C \geq 2$ highlighted in bold) or without fold change criterion (no FCC). R6: backcrossed to C57BL/6 for 6 generations. R1: backcrossed to C57BL/6 for 1 generation. Note that the comparison WT(R6)/APLP2(R6) ${ }^{-/-}$is identical to WT/ $\mathrm{APLP}^{-1-}$.

found for the comparison WT(R6)/APLP2(R6) ${ }^{-/-}$(Figure 9b). Similarly, this also holds true for any of the other pairwise comparisons for which genetic background was kept constant (data not shown).

To study the impact of genetic background more closely, we calculated the percentages of overlap from the most significant 200 probe sets for each of the three comparisons (Figure $8 \mathrm{~b}$ ). If transcriptome changes arise primarily as a consequence of APLP2 deficiency independent of genetic background, we would expect a high number of co-regulated probe sets in the comparisons WT(R6)/APLP2(R6) ${ }^{-1-}$ and WT(R6)/APLP2(R1) $)^{-1-}$. The percentage of probe sets found in this intersection is, however, surprisingly small (3\%). Contrary to our expectation, the highest overlap of probe sets $(47.5 \%)$ is found in the intersection of WT(R6)/APLP2(R1) ${ }^{-/-}$and APLP2 $(\mathrm{R} 6)^{-1-} / \mathrm{APLP} 2(\mathrm{R} 1)^{-1-}$ (Figure 8b).

To gain an overview on absolute number of differentially expressed genes, we created a Venn diagram from the pairwise comparisons WT(R6)/APLP2(R6) $)^{-/-}, \mathrm{WT}$
(R6)/APLP2(R1) ${ }^{-/-}$, and APLP2(R6) $)^{-/-} / \operatorname{APLP} 2(\mathrm{R} 1)^{-/-}(\mathrm{Fig}-$ ure $8 \mathrm{c}$, Additional file 7). Interestingly, we found a set of 27 significant genes in the intersection of WT(R6)/ APLP2(R6) $)^{-1-}$ and APLP2(R6) ${ }^{-1-} /$ APLP2(R1) $)^{-1-}$ (Figure 8c). The corresponding cluster analysis shows that APLP2(R1) ${ }^{-1-}$ samples cluster together with WT(R6) samples while APLP2(R6) ${ }^{-1-}$ samples were clearly separated (Figure 8d). Probe sets in this intersection represent genes that are differentially expressed due to APLP2 deficiency but only in combination with an R6 background. In summary, these findings clearly indicate that genetic background may dominate transcriptome changes and needs to be carefully controlled to establish a clear link between phenotypes and altered genotype.

Eventually, we were interested to identify genes that are highly differentially expressed in our knockout models compared to wild-type. Ccl21 (chemokine (C-C motif) ligand 21) was the gene with the highest fold change in combination with the highest significance score and exclusively up-regulated in APLP2 ${ }^{-1-}$ cortices on R6 genetic background (Figure 10a), that is preferred in behavioral studies and to which we had therefore backcrossed our mutants. In the periphery, CCL21 serves as ligand for CCR7 that is expressed by various cells of the immune systems and is involved in lymphocytes homing (reviewed in [51]). Recent studies show that CCL21 is also expressed in the CNS by endangered neurons to activate microglia via CXCR3 [52,53]. CCL21 is transported in vesicles along the axon to presynaptic structures and thereby constitutes a mediator of directed neuron-microglia signaling and remote microglia activation [54]. As microglia activation is associated with $\mathrm{AD}$ pathogenesis [55] and is also frequently observed as a general indicator of brain damage, we investigated CCL21 expression in more detail. Moreover, we sought to validate the genetic background dependence of
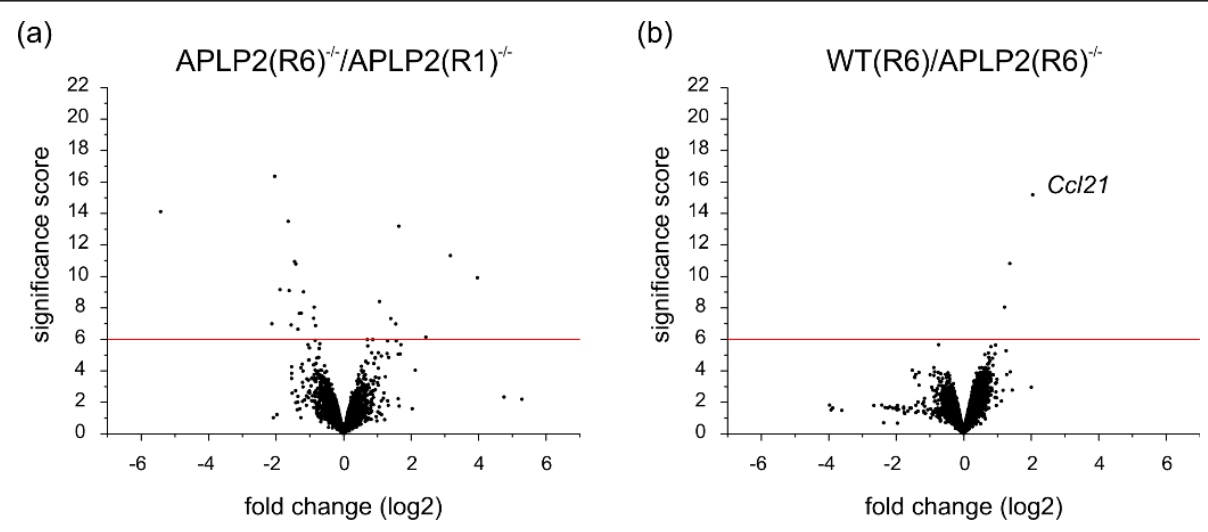

Figure 9 Volcano plot of APLP2(R6) $)^{-/-} /$APLP2(R1) $)^{-/-}$and WT(R6)/APLP2(R6) ${ }^{-/-}$. The absolute value of each probe set identifier's significance score was plotted against the corresponding log2-transformed fold change of (a) APLP2(R6) $)^{-/-} / A P L P 2(R 1)^{-/-}$and (b) WT(R6)/APLP2(R6) ${ }^{-/}$. The red line was set arbitrarily to a score value of 6 to highlight the difference between the two Volcano plots. (APLP2-specific data points were removed prior to plotting). 


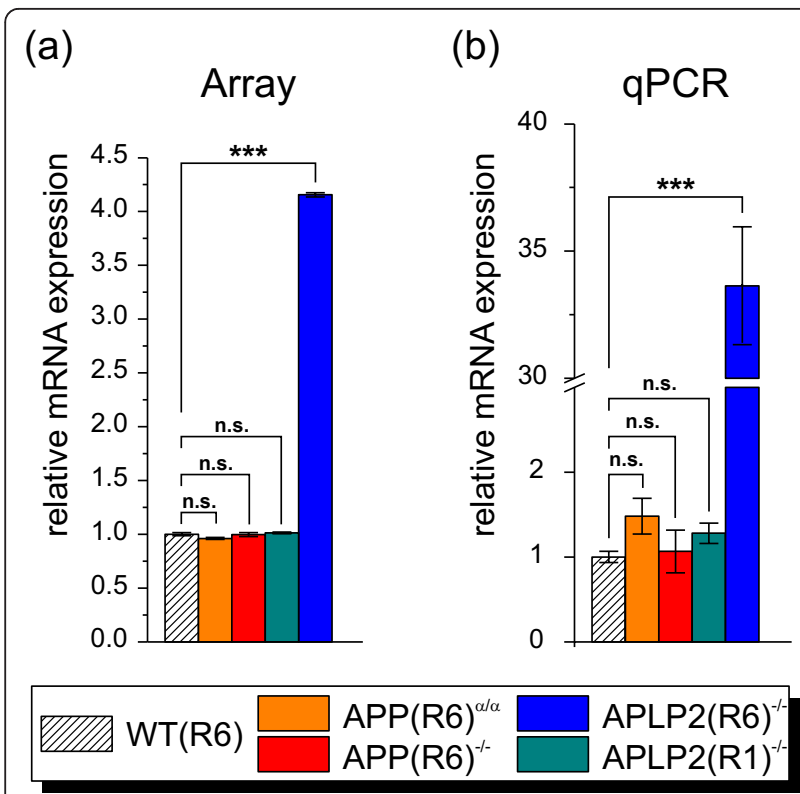

Figure 10 Relative mRNA expression of $C$ cl21. (a) Array-based analysis of Ccl21 mRNA expression normalized to wild-type level. Values represent relative expression value \pm relative standard deviation. Significance was tested by SAM $\left({ }^{*}\right.$, q-value $<0.05 ;{ }^{* *}$, qvalue $<0.01$; ***, q-value $<0.001$ ). (b) qPCR analysis of Ccl21 mRNA expression displayed relative to wild-type level set as one. Values represent means \pm SEM. (Student's t-test, ${ }^{*}, \mathrm{p}$-value $<0.05 ;{ }^{* *}, \mathrm{p}$ value $<0.01{ }^{* * *}, \mathrm{p}$-value $\left.<0.001\right)$.

CCL21 expression. Using qPCR analysis, we confirmed the significant up-regulation of Ccl21 mRNA yielding a fold change in qPCR (33-fold up-regulation) that was even more pronounced compared to array analysis (4fold up-regulation) (Figure 10b). Importantly and consistent with our array analysis, no up-regulation of $C c l 21$ mRNA expression was found in APLP2(R1) ${ }^{-/-}$samples. This indicates that i) other loci distinct from APLP2 are involved in Ccl21 transcriptional regulation and that ii) these loci give rise to allelic variants that differ functionally between the R6 and R1 genetic background. Encouraged by the high increase of Ccl21 mRNA expression, we determined CCL21 protein expression by ELISA in cortical tissue of APLP2(R6) $)^{-1-}$ animals and wild-type controls. CCL21 protein expression was significantly increased by about 1.7-fold (Figure 11a). As this magnitude of up-regulation was lower than expected from qPCR (Figure 10b), we also measured mRNA levels of $C c l 21$ in the same brain samples used for the ELISA measurement and reconfirmed the high up-regulation of about 33-fold (Figure 11b). This difference in differential mRNA (33-fold) and protein (1.7-fold) expression suggests that additional posttranslational mechanisms (including e.g. protein stability and turnover) limit CCL21 expression. In line with the moderate induction of CCL21 protein expression we did not detect an

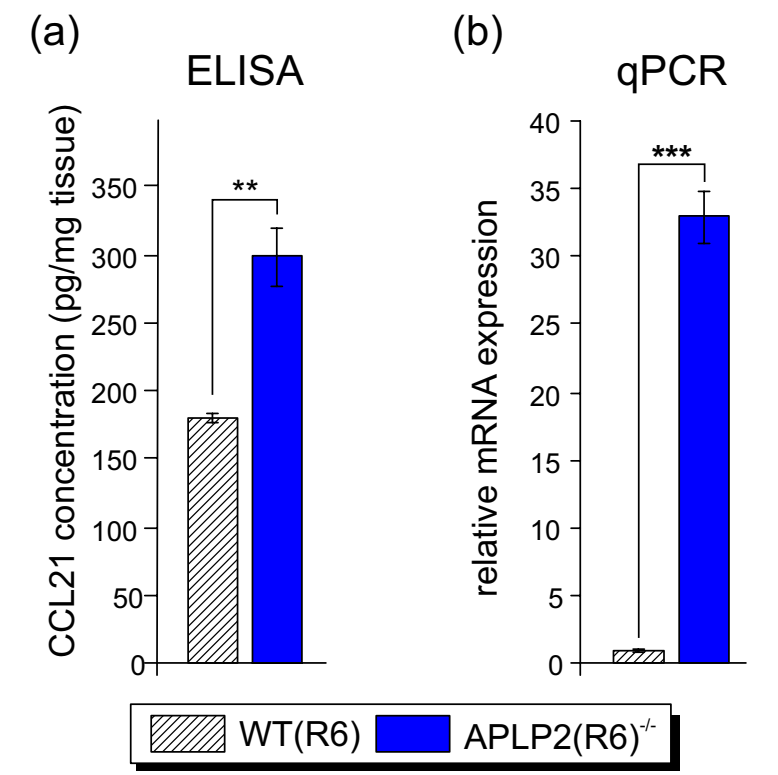

Figure 11 Analysis of CCL21 protein and Ccl21 mRNA expression. (a) CCL21 protein expression was determined by ELISA in either WT(R6) $(n=3)$ or APLP2(R6) $)^{-/-}(n=4)$ brain tissue from one hemisphere comprising cortex, hippocampus and olfactory bulb. (b) qPCR analysis of C 121 mRNA expression using brain tissue from the contralateral side of the same animals (identical brain regions as in (a). (Student's t-test;, p-value $<0.05{ }^{* *}, p$-value $<0.01 ;{ }^{* * *}, p$-value $<0.001)$.

increase in gliosis in APLP2-deficient brains using immunohistochemistry (data not shown).

\section{Conclusions}

Here, we determined the effect of $\mathrm{APP}^{-/-}, \mathrm{APP}^{\alpha / \alpha}$ and $\mathrm{APLP}^{-/-}$genotypes on gene expression in the adult murine cortex. We found large sets of differentially expressed genes, however, fold changes were in most cases only small to moderate. Previously proposed AICD target genes were not convincingly affected by lack of either APP or APLP2 (and thus lack of AICD and ALID) in the complex cortical tissue of adult brain. This may either indicate that the role of AICD in transcriptional regulation has been overestimated or that gene expression changes occur only in a distinct subset of cells that is below the detection level of our analysis.

Remarkably, we found the largest set of differentially expressed genes in APLP2 $2^{-/-}$brain, although so far no apparent morphological or other phenotypic changes had been reported for APLP2-KO mice. A substantial proportion of genes were identified as co-regulated by lack of APP or APLP2, notably in pathways such as neuronal differentiation, neurogenesis and transcriptional regulation. This common genetic profile points towards shared physiological functions in these pathways. When comparing APPs $\alpha$ knockin mice and $\mathrm{APP}^{-/-}$mice we 
observed a close resemblance of the two genotypes pointing towards a crucial role of the APP C-terminus for transcriptome changes. Interestingly, we could demonstrate that several synaptic plasticity-related genes found in this gene set are considerably down-regulated which further substantiates the importance of APP family members in this regard.

Finally, we addressed the role of genetic background for transcriptome changes. Here, we report that the presence of different WT-alleles can lead to profound changes in gene expression that are even higher in magnitude than those resulting from the knockout of a single gene such as APLP2. Thus, it is crucial to keep genetic background constant, particularly if gene expression changes are rather subtle to reliably correlate affected pathways (and physiological functions inferred from them) with a knockout phenotype. In many studies regarding AICD signaling this issue has not been addressed which may at least partially explain the conflicting results reported by different laboratories. Here, we identified the chemokine $C c l 21$ as a gene that is highly up-regulated in APLP2 ${ }^{-/}$cortex, but only in conjunction with C57BL/6-specific background alleles. Moreover, our study corroborates that APP family members are not only structurally related but also serve related physiological functions. It will therefore be of high interest to analyze phenotypic and gene expression changes in adult APP/APLP2 double or APP/APLP1/APLP2 triple deficient brain, once viable conditional combined mutants become available that are currently generated by crossing mice with floxed APP and APLP2 alleles with transgenic tissue-specific Cre mice [56].

\section{Methods \\ Data}

Raw and processed data discussed in this publication have been deposited in the NCBI's Gene Expression Omnibus database (GEO) and are accessible through GEO Series accession number GSE25926 (http://www. ncbi.nlm.nih.gov/geo/query/acc.cgi?acc=GSE25926).

\section{Animals}

$\mathrm{APP}^{-1-}, \mathrm{APLP}^{-1-}, \mathrm{APP}^{\alpha / \alpha}$ animals were previously described $[28,57,58]$. All animals were kept under specific pathogen free housing conditions (SPF unit) and in compliance with the regulations of the German animal protection law. For transcriptome analysis, animals had been backcrossed to C57BL/6 wild-type animals for 6 generations (R6) before they were interbred to homozygosity. All animals were adult males (24-28 weeks) and not challenged with any cognitive or stress tasks.

\section{RNA preparation and microarray data generation}

Animals were sacrificed by cervical dislocation. Mouse brains were dissected and stored in RNAlater (Qiagen) at $-20^{\circ} \mathrm{C}$. Subsequently, the prefrontal cortex was cut out and used for total RNA preparation (RNAeasy kit, Qiagen). Quality of RNA was assessed with a spectrophotometer and Bioanalyzer (Agilent). $1 \mu \mathrm{g}$ of total RNA was used for cDNA preparation (Oligo(dT) method, Invitrogen). Subsequent cRNA was prepared with Affymetrix One-Cycle Target Labeling and Control Reagent kit (Affymetrix Inc., Santa Clara, California, USA). The biotinylated cRNA was hybridized onto GeneChip Mouse Genome 4302.0 Arrays (Affymetrix, Santa Clara). Chips were washed and scanned on the Affymetrix Complete GeneChip ${ }^{\circledR}$ Instrument System generating digitized image data files.

\section{Statistical analysis}

If not stated otherwise, data analysis and processing was carried out within the statistical computing environment $\mathrm{R}$, version 2.8.0, using Bioconductor, BioC Release 2.4 [59]. Raw data was processed with the RMA algorithm (Robust Multiarray Average) developed by Irizarry et al. [29] and normalized using quantile normalization [30].

Hierarchical clustering was carried out using Euclidean distances to calculate the distances between the genes and between the sample groups. Calculated distances were clustered by complete linkage clustering. Expression values for each probe set were normalized to zero mean and unit variance. The values shown thus represent the number of standard deviations above or below the mean expression for each gene. Calculated expression differences for each probe set can be found in the respective additional file.

Significant differentially expressed probe sets between two groups were detected by a Significance Analysis of Microarrays (SAM) [60]. As a cut-off value for significance, we set the false discovery rate (FDR) to $5.33 \%$ $\left(\mathrm{WT}_{\mathrm{APP}}{ }^{-/}\right), 4.96 \%\left(\mathrm{WT} / \mathrm{APLP}^{-1-}\right), 4.5 \%\left(\mathrm{WT} / \mathrm{APP}^{\alpha / \alpha}\right)$, $4.79 \%\left(\mathrm{APP}^{\alpha / \alpha} / \mathrm{APP}^{-/-}\right), 5.02 \%\left(\mathrm{WT}(\mathrm{R} 6) / \mathrm{APLP} 2(\mathrm{R} 1)^{-/-}\right)$, and $5.11 \%$ (APLP2(R6) $)^{-/-} /$APLP2(R1) $)^{-/-}$).

For counting significant differentially expressed genes, probe set identifiers were mapped to Entrez Gene identifiers. If at least one probe set was significant in the SAM, the gene was regarded to be significant as well. If no gene information (Entrez ID) was available for a certain probe set, the probe set was not counted.

For group testing (GO terms, pathways) DAVID bioinformatics resources was used [61]. Gene symbols from each list were taken as input, and redundant entries were discarded. The following gene sets were included into the analysis: GOTERM_BP_FAT (Gene Ontology), Biocarta (Pathways), KEGG_PATHWAY, PANTHER_PATHWAY, REACTOME_PATHWAY. Functional annotation clustering was carried out using the highest classification stringency. 


\section{Quantitative real-time PCR (qPCR)}

Total RNA was prepared using High Capacity cDNA kit based on random hexamer primer method (Applied Biosystems). For each qPCR reaction 20ng of total RNA were reverse transcribed into cDNA. qPCR was performed using FAM $^{\mathrm{TM}}$-MGB dye labeled TaqMan ${ }^{\circledR}$ Gene Expression Assays (Applied Biosystems) for Bace1 (assay Mm00478664_m1), Kai1 (assay Mm00492061_m1), Egfr (assay Mm01187858_m1), Gsk3b (assay Mm00444911_m1), p53 (assay Mm01731290_g1), Tip60 (assay Mm00724374_m1), Vglut2 (assay Mm00499876_m1), Hspa5 (assay Mm00517690_g1), Cdkn1a/p21 (assay Mm01303209_m1), Arc (assay Mm00479619_g1), Fos (assay Mm00487426_g1), Egr2 (assay Mm00456650_m1), Dio2 (assay Mm00515664_m1), Ccl21 (assay Mm03646971_gH) and beta-Actin as an internal standard (assay 4352933E). Quantification of qPCR results were evaluated by the $2^{-}$ $\triangle \triangle C T$ method and normalized to wild-type animals. Significance was calculated using unpaired Student's t-test $\left({ }^{*}, \mathrm{p}<0.05 ;{ }^{* * *}, \mathrm{p}<0.01 ;{ }^{* * * *}, \mathrm{p}<0.001\right)$.

\section{CCL21 ELISA measurements}

Brain homogenates for ELISA were generated as described before [62]. Briefly brains were homogenized in lysis buffer (100mM phosphate, $\mathrm{pH} 7.4,1 \mathrm{mM}$ EDTA, supplemented with complete protease inhibitor cocktail (Roche, Germany) using a PotterS homogenizer (Sartorius, Germany), followed by centrifugation at 1,500 $\times \mathrm{g}$ for $10 \mathrm{~min}$. Supernatants were directly used for ELISA determinations.

ELISA measurements were performed using a mouse CCL21/6Ckine kit ( $\mathrm{R} \& \mathrm{D}$ Systems Inc., MN) according to the manufacturer's protocol with slight modifications. Briefly, the standard curve was performed in a concentration range of $0-5000 \mathrm{pg} / \mathrm{ml}$, the antibodies were used in the dilutions suggested by the protocol, except for the HRP-streptavidine conjugate, which was diluted 1:100. As a substrate one-step TMB-ELISA (Thermo Scientific, IL) was used.

\section{Additional material}

Additional file 1: Heatmap of the processed dataset. The heatmap shows the clustering of the processed data by App/Ap/p2-specific probe sets. App and Aplp2 probe sets were taken from the ENSEMBL database and remapped onto the modified respective genomic loci of $\mathrm{APP}^{-1}$ $\mathrm{APP}^{\alpha / \alpha}$, and $\mathrm{APLP}^{-/-}$animals. Only probe sets that map to exonic sequences or UTRs were chosen for hierarchical cluster analysis. The first three probe sets correspond to App probe sets whereas the last five are Aplp2-specific. The values of the heatmap are normalized expression values with red and blue color representing the number of standard standard deviations above or below the mean expression for each probe set, respectively.

Additional file 2: Lists of significant probe sets (R6 animals) resulting from the pairwise significance analyses. for each significant
Affymetrix probe set identifier, information about the gene (ENTREZ gene ID, gene symbol, gene name) and the output from the SAM (score, numerator, denominator, fold change, q-value) are displayed. Data was ranked by the absolute test score. WT/APP ${ }^{-/-}(=$WTAPP $)$, WT $/ A P L P 2^{-/-}(=$ WTAPLP2), WT/APP ${ }^{\alpha / \alpha}$ (= WTAPPsa), APP $^{\alpha / \alpha} / \mathrm{APP}^{-/}$(= APPsaAPP).

Additional file 3: Result from the GO term and pathway analysis using DAVID for WT/APP ${ }^{-/}$, WT/APP ${ }^{\alpha / \alpha}$, WT/APLP2 ${ }^{-/-}$. Annotation clusters are displayed with their respective enrichment score. For each annotation cluster, the gene groups ( $G O$ terms, pathways) that belong to the cluster are listed including test statistics and genes in the gene group that were significant in the respective pairwise comparison (WT/ $\mathrm{APP}^{-1-}$ (= WTAPP), WT/APP ${ }^{\alpha / \alpha}$ (WTAPPsa), WT/APLP2 ${ }^{-1-}$ (= WTAPLP2)).

Additional file 4: Lists of probe sets in the intersections of WT/ APP $^{-/-}$, WT/APLP2 ${ }^{-/-}$, and WT/APP ${ }^{\alpha / \alpha}$. for each significant Affymetrix probe set identifier, information about the gene (ENTREZ gene ID, gene symbol, gene name), the output from the SAM (score, numerator, denominator, fold change, q-value), and the respective pairwise comparison are displayed for the intersection of WT/APP ${ }^{-/}$and WT/ $\mathrm{APLP}^{-1-}$ (= WTAPP \& WTAPLP2) as well as the intersection of WT/APP ${ }^{-/}$ and WT/APLP2 $2^{-/-}$and WT/APP ${ }^{\alpha / \alpha}$ (= WTAPP \& WTAPLP2 \& WTAPPsa). Data was ranked by gene symbols.

Additional file 5: Lists of probe set in the intersections of WT/APP ${ }^{-/}$, WT/APP ${ }^{\alpha / \alpha}$, APP $^{\alpha / \alpha} / \mathrm{APP}^{-/-}$. for each significant Affymetrix probe set identifier, information about the gene (ENTREZ gene ID, gene symbol, gene name), the output from the SAM (score, numerator, denominator, fold change, $q$-value), and the respective pairwise comparison are displayed for the intersection of WT/APP ${ }^{-/-}$and WT/APP ${ }^{\alpha / \alpha}$ (= WTAPP \& WTAPPsa) as well as WT/APP ${ }^{-/}$and APP AP $^{\alpha / \alpha} /$ APP $^{-1}$ (= WTAPP \& APPsaAPP) and WT/APP ${ }^{\alpha / \alpha}$ and APP ${ }^{\alpha / \alpha} / \mathrm{APP}^{-/-}(=$WTAPPsa \& APPsaAPP). Data was ranked by gene symbols.

Additional file 6: Lists of significant probe sets of animals with mixed genetic background compared to backcrossed animals. Results from the pairwise significance analyses: WT(R6)/APLP2(R1) $)^{-/-}(=\mathrm{WT}$ (R6)APLP2(R1)), APLP2(R6) ${ }^{-/} / A P L P 2(R 1)^{-/-}$(= APLP2(R6)APLP2(R1)). For each significant Affymetrix probe set identifier, information about the gene (ENTREZ gene ID, gene symbol, gene name) and the output from the SAM (score, numerator, denominator, fold change, q-value) are displayed. Data was ranked by the absolute test score.

Additional file 7: Lists of probe set in the intersections of WT(R6)/

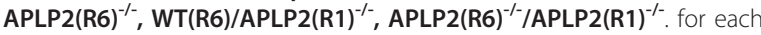
significant Affymetrix probe set identifier, information about the gene (ENTREZ gene ID, gene symbol, gene name) and the output from the SAM (score, numerator, denominator, fold change, q-value), and the respective pairwise comparison are displayed for the intersection of WT $(\mathrm{R} 6) / \mathrm{APLP} 2(\mathrm{R} 1)^{-/-}$and APLP2(R6) $)^{-/} / \mathrm{APLP} 2(\mathrm{R} 1)^{-/-}$(= WTAPLP2(R1) \& R6R1), WT(R6)/APLP2(R6) $)^{-/-}$and APLP2(R6) $/$/APLP2(R1) ( $^{-/-}$(= WTAPLP2 \& R6R1), and WT/APLP2 ${ }^{-/}$and WT/APLP2(R1) ${ }^{-/-}(=$WTAPLP2 \& WTAPLP2(R1)). Data was ranked by gene symbols.

\section{Acknowledgements}

We thank J. Gobbert and M. Saile for excellent technical assistance. This work was supported by grants from the NGFNplus, Deutsche Forschungsgemeinschaft (SFB488-D18, MU1457/8-1 and MU1457/9-1) and the Hans and Ilse Breuer foundation to UCM.

\section{Author details}

${ }^{1}$ Department of Bioinformatics and Functional Genomics, Institute of Pharmacy and Molecular Biotechnology, Heidelberg University, Im Neuenheimer Feld 364, D-69120 Heidelberg, Germany. ${ }^{2}$ Medical Research Center, Medical Faculty Mannheim, Heidelberg University, D-68167 Mannheim, Germany. ${ }^{3}$ Institute for Neuropathology, University Hospital Freiburg, Freiburg, Germany. ${ }^{4}$ Department of Theoretical Bioinformatics, German Cancer Research Center (DKFZ), D-69120 Heidelberg, Germany.

\section{Authors' contributions}

All authors read and approved the final manuscript. DA performed the bioinformatics analysis and drafted the manuscript. MAF harvested the 
tissue, performed all molecular genetics experiments and co-drafted the manuscript. JAT performed ELISA experiments. NG coordinated and supervised microarray hybridization and data acquisition. MP performed immunohistochemistry of brain sections to assess gliosis. RE coordinated the bioinformatics analysis. BB participated in study design, supervised the bioinformatics analysis and contributed to drafting the manuscript. UCM conceived and designed the study and drafted the final version of the manuscript.

Received: 22 December 2010 Accepted: 24 March 2011

Published: 24 March 2011

\section{References}

1. Kimberly WT, Zheng JB, Guenette SY, Selkoe DJ: The intracellular domain of the beta-amyloid precursor protein is stabilized by Fe65 and translocates to the nucleus in a notch-like manner. J Biol Chem 2001, 276:40288-40292.

2. Cao X, Sudhof TC: A transcriptionally [correction of transcriptively] active complex of APP with Fe65 and histone acetyltransferase Tip60. Science 2001, 293:115-120

3. Gao Y, Pimplikar SW: The gamma -secretase-cleaved C-terminal fragment of amyloid precursor protein mediates signaling to the nucleus. Proceedings of the National Academy of Sciences of the United States of America 2001, 98:14979-14984.

4. Scheinfeld MH, Ghersi E, Laky K, Fowlkes BJ, D'Adamio L: Processing of beta-amyloid precursor-like protein- 1 and -2 by gamma-secretase regulates transcription. J Biol Chem 2002, 277:44195-44201.

5. Swistowski A, Zhang Q, Orcholski ME, Crippen D, Vitelli C, Kurakin A Bredesen DE: Novel mediators of amyloid precursor protein signaling. J Neurosci 2009, 29:15703-15712.

6. Orcholski ME, Zhang Q, Bredesen DE: Signaling via Amyloid Precursor-Like Proteins APLP1 and APLP2. J Alzheimers Dis 2010.

7. Baek SH, Ohgi KA, Rose DW, Koo EH, Glass CK, Rosenfeld MG: Exchange of $\mathrm{N}-\mathrm{CoR}$ corepressor and Tip60 coactivator complexes links gene expression by NF-kappaB and beta-amyloid precursor protein. Cell 2002, 110:55-67.

8. Kim HS, Kim EM, Lee JP, Park CH, Kim S, Seo JH, Chang KA, Yu E, Jeong SJ, Chong $\mathrm{YH}$, Suh $\mathrm{YH}$ : C-terminal fragments of amyloid precursor protein exert neurotoxicity by inducing glycogen synthase kinase-3beta expression. Faseb J 2003, 17:1951-1953.

9. Ryan KA, Pimplikar SW: Activation of GSK-3 and phosphorylation of CRMP2 in transgenic mice expressing APP intracellular domain. J Cell Biol 2005, 171:327-335.

10. Pardossi-Piquard R, Petit A, Kawarai T, Sunyach C, Alves da Costa C, Vincent B, Ring S, D'Adamio L, Shen J, Muller U, et al: Presenilin-dependent transcriptional control of the Abeta-degrading enzyme neprilysin by intracellular domains of betaAPP and APLP. Neuron 2005, 46:541-554.

11. Zhang YW, Wang R, Liu Q, Zhang H, Liao FF, Xu H: Presenilin/gammasecretase-dependent processing of beta-amyloid precursor protein regulates EGF receptor expression. Proceedings of the National Academy of Sciences of the United States of America 2007, 104:10613-10618.

12. Alves da Costa C, Sunyach C, Pardossi-Piquard R, Sevalle J, Vincent B, Boyer N, Kawarai T, Girardot N, St George-Hyslop P, Checler F: Presenilindependent gamma-secretase-mediated control of p53-associated cell death in Alzheimer's disease. J Neurosci 2006, 26:6377-6385.

13. Liu Q, Zerbinatti CV, Zhang J, Hoe HS, Wang B, Cole SL, Herz J, Muglia L, $\mathrm{Bu}$ G: Amyloid precursor protein regulates brain apolipoprotein $\mathrm{E}$ and cholesterol metabolism through lipoprotein receptor LRP1. Neuron 2007, 56:66-78.

14. von Rotz RC, Kohli BM, Bosset J, Meier M, Suzuki T, Nitsch RM, Konietzko U: The APP intracellular domain forms nuclear multiprotein complexes and regulates the transcription of its own precursor. Journal of cell science 2004, 117:4435-4448.

15. Muller T, Concannon CG, Ward MW, Walsh CM, Tirniceriu AL, Tribl F, Kogel D, Prehn JH, Egensperger R: Modulation of gene expression and cytoskeletal dynamics by the amyloid precursor protein intracellular domain (AICD). Molecular biology of the cell 2007, 18:201-210.

16. Hebert SS, Serneels L, Tolia A, Craessaerts K, Derks C, Filippov MA, Muller U, De Strooper B: Regulated intramembrane proteolysis of amyloid precursor protein and regulation of expression of putative target genes. EMBO Rep 2006, 7:739-745.
17. Yang Z, Cool BH, Martin GM, Hu Q: A dominant role for FE65 (APBB1) in nuclear signaling. J Biol Chem 2006, 281:4207-4214.

18. Chen AC, Selkoe DJ: Response to: Pardossi-Piquard et al., "PresenilinDependent Transcriptional Control of the Abeta-Degrading Enzyme Neprilysin by Intracellular Domains of betaAPP and APLP." Neuron 46, 541-554. Neuron 2007, 53:479-483.

19. Repetto E, Yoon IS, Zheng H, Kang DE: Presenilin 1 regulates epidermal growth factor receptor turnover and signaling in the endosomallysosomal pathway. J Biol Chem 2007, 282:31504-31516.

20. Giliberto L, Zhou D, Weldon R, Tamagno E, De Luca P, Tabaton M, D'Adamio L: Evidence that the Amyloid beta Precursor Proteinintracellular domain lowers the stress threshold of neurons and has a "regulated" transcriptional role. Molecular neurodegeneration 2008, 3:12.

21. Tamboli IY, Prager K, Thal DR, Thelen KM, Dewachter I, Pietrzik CU, St George-Hyslop P, Sisodia SS, De Strooper B, Heneka MT, et al: Loss of gamma-secretase function impairs endocytosis of lipoprotein particles and membrane cholesterol homeostasis. J Neurosci 2008, 28:12097-12106

22. Waldron E, Isbert S, Kern A, Jaeger S, Martin AM, Hebert SS, Behl C, Weggen S, De Strooper B, Pietrzik CU: Increased AICD generation does not result in increased nuclear translocation or activation of target gene transcription. Exp Cell Res 2008, 314:2419-2433.

23. Magara F, Muller U, Li ZW, Lipp HP, Weissmann C, Stagljar M, Wolfer DP: Genetic background changes the pattern of forebrain commissure defects in transgenic mice underexpressing the beta-amyloid-precursor protein. Proceedings of the National Academy of Sciences of the United States of America 1999, 96:4656-4661.

24. Heber S, Herms J, Gajic V, Hainfellner J, Aguzzi A, Rulicke T, von Kretzschmar H, von Koch C, Sisodia S, Tremml P, et al: Mice with combined gene knock-outs reveal essential and partially redundant functions of amyloid precursor protein family members. J Neurosci 2000, 20:7951-7963.

25. Herms J, Anliker B, Heber S, Ring S, Fuhrmann M, Kretzschmar H, Sisodia S, Muller U: Cortical dysplasia resembling human type 2 lissencephaly in mice lacking all three APP family members. The EMBO journal 2004, 23:4106-4115.

26. Anliker B, Muller $\mathrm{U}$ : The functions of mammalian amyloid precursor protein and related amyloid precursor-like proteins. Neurodegener Dis 2006, 3:239-246.

27. Taylor CJ, Ireland DR, Ballagh I, Bourne K, Marechal NM, Turner PR, Bilkey DK, Tate WP, Abraham WC: Endogenous secreted amyloid precursor protein-alpha regulates hippocampal NMDA receptor function, long-term potentiation and spatial memory. Neurobiol Dis 2008, 31:250-260.

28. Ring S, Weyer SW, Kilian SB, Waldron E, Pietrzik CU, Filippov MA, Herms J, Buchholz C, Eckman CB, Korte M, et al: The secreted beta-amyloid precursor protein ectodomain APPs alpha is sufficient to rescue the anatomical, behavioral, and electrophysiological abnormalities of APPdeficient mice. J Neurosci 2007, 27:7817-7826.

29. Irizarry RA, Hobbs B, Collin F, Beazer-Barclay YD, Antonellis KJ, Scherf U, Speed TP: Exploration, normalization, and summaries of high density oligonucleotide array probe level data. Biostatistics (Oxford, England) 2003, 4:249-264.

30. Bolstad BM, Irizarry RA, Astrand M, Speed TP: A comparison of normalization methods for high density oligonucleotide array data based on variance and bias. Bioinformatics (Oxford, England) 2003, 19:185-193.

31. Prinzen C, Trumbach D, Wurst W, Endres K, Postina R, Fahrenholz F: Differential gene expression in ADAM10 and mutant ADAM10 transgenic mice. BMC genomics 2009, 10:66

32. Huang da W, Sherman BT, Lempicki RA: Systematic and integrative analysis of large gene lists using DAVID bioinformatics resources. Nat Protoc 2009, 4:44-57.

33. Hayashi Y, Kashiwagi K, Ohta J, Nakajima M, Kawashima T, Yoshikawa K: Alzheimer amyloid protein precursor enhances proliferation of neural stem cells from fetal rat brain. Biochem Biophys Res Commun 1994, 205:936-943.

34. Ohsawa I, Takamura C, Morimoto T, Ishiguro M, Kohsaka S: Amino-terminal region of secreted form of amyloid precursor protein stimulates proliferation of neural stem cells. Eur J Neurosci 1999, 11:1907-1913.

35. Caille I, Allinquant B, Dupont E, Bouillot C, Langer A, Muller U, Prochiantz A: Soluble form of amyloid precursor protein regulates proliferation of 
progenitors in the adult subventricular zone. Development 2004, 131:2173-2181

36. Crews L, Rockenstein E, Masliah E: APP transgenic modeling of Alzheimer's disease: mechanisms of neurodegeneration and aberrant neurogenesis. Brain Struct Funct 2010, 214:111-126.

37. Schrenk-Siemens K, Perez-Alcala S, Richter J, Lacroix E, Rahuel J, Korte M, Muller U, Barde YA, Bibel M: Embryonic stem cell-derived neurons as a cellular system to study gene function: lack of amyloid precursor proteins APP and APLP2 leads to defective synaptic transmission. Stem cells (Dayton, Ohio) 2008, 26:2153-2163.

38. Jacobsen KT, Iverfeldt K: Amyloid precursor protein and its homologues: a family of proteolysis-dependent receptors. Cell Mol Life Sci 2009, 66:2299-2318

39. Yang Y, Turner RS, Gaut JR: The chaperone BiP/GRP78 binds to amyloid precursor protein and decreases Abeta40 and Abeta42 secretion. J Biol Chem 1998, 273:25552-25555.

40. Venkataramani V, Rossner C, Iffland L, Schweyer S, Tamboli IY, Walter J, Wirths O, Bayer TA: Histone deacetylase inhibitor valproic acid inhibits cancer cell proliferation via down-regulation of the alzheimer amyloid precursor protein. J Biol Chem 2010, 285:10678-10689.

41. Hoshino T, Nakaya T, Araki W, Suzuki K, Suzuki T, Mizushima T: Endoplasmic reticulum chaperones inhibit the production of amyloid-beta peptides. Biochem J 2007, 402:581-589.

42. Pechnick RN, Zonis S, Wawrowsky K, Pourmorady J, Chesnokova V: p21Cip1 restricts neuronal proliferation in the subgranular zone of the dentate gyrus of the hippocampus. Proceedings of the National Academy of Sciences of the United States of America 2008, 105:1358-1363.

43. Li H, Wang B, Wang Z, Guo Q, Tabuchi K, Hammer RE, Sudhof TC, Zheng H: Soluble amyloid precursor protein (APP) regulates transthyretin and Klotho gene expression without rescuing the essential function of APP. Proceedings of the National Academy of Sciences of the United States of America 2010, 107:17362-17367.

44. Bramham CR, Worley PF, Moore MJ, Guzowski JF: The immediate early gene arc/arg3.1: regulation, mechanisms, and function. J Neurosci 2008, 28:11760-11767.

45. DeSteno DA, Schmauss C: Induction of early growth response gene 2 expression in the forebrain of mice performing an attention-set-shifting task. Neuroscience 2008, 152:417-428.

46. Fleischmann A, Hvalby O, Jensen V, Strekalova T, Zacher C, Layer LE, Kvello A, Reschke M, Spanagel R, Sprengel R, et al: Impaired long-term memory and NR2A-type NMDA receptor-dependent synaptic plasticity in mice lacking c-Fos in the CNS. J Neurosci 2003, 23:9116-9122.

47. Guzowski JF: Insights into immediate-early gene function in hippocampal memory consolidation using antisense oligonucleotide and fluorescent imaging approaches. Hippocampus 2002, 12:86-104.

48. Poirier R, Cheval H, Mailhes C, Charnay P, Davis S, Laroche S: Paradoxical role of an Egr transcription factor family member, Egr2/Krox20, in learning and memory. Front Behav Neurosci 2007, 1:6.

49. Wolfer DP, Lipp HP: Dissecting the behaviour of transgenic mice: is it the mutation, the genetic background, or the environment? Exp Physiol 2000, 85:627-634.

50. Ryman D, Lamb BT: Genetic and environmental modifiers of Alzheimer's disease phenotypes in the mouse. Curr Alzheimer Res 2006, 3:465-473.

51. Forster R, Davalos-Misslitz AC, Rot A: CCR7 and its ligands: balancing immunity and tolerance. Nat Rev Immunol 2008, 8:362-371.

52. Biber K, Sauter A, Brouwer N, Copray SC, Boddeke HW: Ischemia-induced neuronal expression of the microglia attracting chemokine Secondary Lymphoid-tissue Chemokine (SLC). Glia 2001, 34:121-133.

53. Rappert A, Biber K, Nolte C, Lipp M, Schubel A, Lu B, Gerard NP, Gerard C, Boddeke HW, Kettenmann H: Secondary lymphoid tissue chemokine (CCL21) activates CXCR3 to trigger a Cl- current and chemotaxis in murine microglia. J Immunol 2002, 168:3221-3226.

54. de Jong EK, Dijkstra IM, Hensens M, Brouwer N, van Amerongen M, Liem RS, Boddeke HW, Biber K: Vesicle-mediated transport and release of CCL21 in endangered neurons: a possible explanation for microglia activation remote from a primary lesion. J Neurosci 2005, 25:7548-7557.

55. Akiyama H, Barger S, Barnum S, Bradt B, Bauer J, Cole GM, Cooper NR, Eikelenboom P, Emmerling M, Fiebich BL, et al: Inflammation and Alzheimer's disease. Neurobiology of aging 2000, 21:383-421.

56. Mallm JP, Tschape JA, Hick M, Filippov MA, Muller UC: Generation of conditional null alleles for APP and APLP2. Genesis 2010, 48:200-206.
57. Li ZW, Stark G, Gotz J, Rulicke T, Gschwind M, Huber G, Muller U, Weissmann C: Generation of mice with a 200-kb amyloid precursor protein gene deletion by Cre recombinase-mediated site-specific recombination in embryonic stem cells. Proceedings of the National Academy of Sciences of the United States of America 1996, 93:6158-6162.

58. von Koch CS, Zheng H, Chen H, Trumbauer M, Thinakaran G, van der Ploeg LH, Price DL, Sisodia SS: Generation of APLP2 KO mice and early postnatal lethality in APLP2/APP double KO mice. Neurobiology of aging 1997, 18:661-669.

59. Gentleman RC, Carey VJ, Bates DM, Bolstad B, Dettling M, Dudoit S, Ellis B, Gautier L, Ge Y, Gentry J, et al: Bioconductor: open software development for computational biology and bioinformatics. Genome biology 2004, 5 : R80.

60. Tusher VG, Tibshirani R, Chu G: Significance analysis of microarrays applied to the ionizing radiation response. Proceedings of the National Academy of Sciences of the United States of America 2001, 98:5116-5121.

61. Dennis G Jr, Sherman BT, Hosack DA, Yang J, Gao W, Lane HC, Lempicki RA: DAVID: Database for Annotation, Visualization, and Integrated Discovery. Genome biology 2003, 4:P3.

62. Zhao P, Waxman SG, Hains BC: Modulation of thalamic nociceptive processing after spinal cord injury through remote activation of thalamic microglia by cysteine cysteine chemokine ligand 21. J Neurosci 2007, 27:8893-8902.

\section{doi:10.1186/1471-2164-12-160}

Cite this article as: Aydin et al: Comparative transcriptome profiling of amyloid precursor protein family members in the adult cortex. BMC Genomics 2011 12:160.

\section{Submit your next manuscript to BioMed Central and take full advantage of:}

- Convenient online submission

- Thorough peer review

- No space constraints or color figure charges

- Immediate publication on acceptance

- Inclusion in PubMed, CAS, Scopus and Google Scholar

- Research which is freely available for redistribution

Submit your manuscript at www.biomedcentral.com/submit
Biomed Central 